\title{
Time-Synchronized Laser-Induced Fluorescence in the Near-Field of a 600 Watt Hall Thruster
}

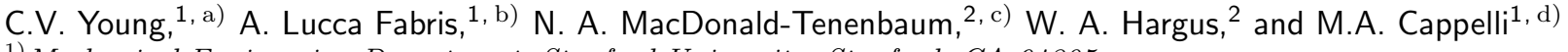 \\ 1) Mechanical Engineering Department, Stanford University, Stanford, CA 94305 \\ 2) Edwards Air Force Base, CA 93524, USA
}

(Dated: 21 September 2021)

We report on the results of an experimental campaign to measure time-varying velocity distributions in the near-field of a low power Hall thruster. We employ a sample-hold technique, enhanced by parallelizing the measurement hardware into several signal processing channels that vastly increases the data acquisition rate. The measurements are applied to study flow field dynamics in a commercial BHT-600 Hall thruster undergoing unforced breathing mode oscillations in the $44-49 \mathrm{kHz}$ range. A very detailed experimental picture of the nearfield emerges from these studies. The results indicate that velocity fluctuations lessen further downstream of the exit plane. Along the thruster axis, where there is a general appearance of a central jet, there is evidence of a low velocity ion population in between the periodic bursts of high velocity ions, indicative of local ionization of neutrals outside of the thruster. One possible source of this residual ionization may be background chamber gas, which is not unexpected with the limited pumping capacity of ground test facilities.

Keywords: time-resolved laser-induced fluorescence, plasma diagnostics, electric propulsion, Hall thruster, breathing mode, plasma oscillation

\section{INTRODUCTION}

The Hall Effect Thruster ${ }^{1,2}$ (HET) has become a predominant plasma-based electric propulsion technology now used on several spacecrafts. The fundamental operating physics of these devices has been the topic of considerable research during the past few decades. The core of this technology relies on a direct-current magnetized plasma discharge created in an annular ceramiclined channel that is open at one end. The anode of the system, through which propellant (usually xenon or krypton) is introduced, is located at the closed end of the channel while the cathode (usually a hollow cathode with a thermionic electron emitter) is mounted externally to the channel. A potential difference is applied between the anode and cathode that supports the discharge and a radial magnetic field is applied across the channel just upstream of the exit, partially trapping electrons in a closed azimuthal drift. A consequence of this is a strong axial electric field concentration which acts to accelerate the largely non-magnetized ions out of the channel to velocities in the range of $\sim 20000 \mathrm{~m} / \mathrm{s}$. The cathode serves to provide the electrons needed to sustain the plasma discharge in addition to those needed to neutralize the ensuing ion beam.

Despite the maturity and extensive flight heritage of this technology, accurate modeling and predictions of

\footnotetext{
a) Presently at Lawrence Livermore National Laboratory, 7000 East Ave., Livermore, CA 94550, USA; Electronic mail: cvyoung@alumni.stanford.edu

b) Presently at Surrey Space Centre, University of Surrey, Guildford GU2 7XH, UK

c) Presently at Aerojet Rocketdyne, Redmond, WA 98052, USA

d) http://www.sppl.stanford.edu
}

performance under flight conditions remain a challenge. The investigation of many specific phenomena that influence thruster performance and operation is still a relevant topic of contemporary research while a complete fundamental understanding is lacking. One such open research question concerns the solution of the physics governing the plethora of broadband instabilities and oscillations that characterize Hall discharges under certain operating conditions. One of the strongest oscillatory regimes is the so-called breathing mode, ${ }^{3,4}$ with typical fundamental harmonics in the $10-50 \mathrm{kHz}$ range, sustained by alternating cycles of propellant ionization, expulsion of ions, and replenishment of neutrals within the discharge channel. This phenomenon has been described to a first approximation by means of a predatorprey type relationship ${ }^{5,6}$ between the neutral propellant and ions released behind a traveling ionization front, and more refined models have captured a complex interplay between the motion of the ionization front, the evolution of the potential topology established in the channel, and the acceleration of ions during breathing mode oscillations. From a phenomenological aspect, the device operation under breathing mode oscillations manifests intense discharge current fluctuations with a sometimes quasi-periodic nature (i.e. a Fourier spectrum relatively stable in time). The time-resolved experimental measurement of the plasma properties during a discharge current cycle is paramount for giving a direct contribution to the understanding of the governing physics. Both invasive plasma probes ${ }^{7-11}$ and noninvasive optical diagnostic methods have been applied to this purpose. The realm of the latter category includes time-resolved optical emission studies ${ }^{12-14}$ and novel time-resolved laserinduced fluorescence (LIF) techniques. ${ }^{15,16}$

Multiple strategies ${ }^{15,17-20}$ have been developed for mapping Hall thruster ion velocities in the combined time 
and space domains, capturing the time evolution of the local ion velocity distribution function (IVDF). The various techniques present different signal processing features achieving finer resolutions in either the ion velocity dimension or the time dimension. Some of them are able to resolve natural oscillations within the discharge plasma, while others rely on artificial excitation of the oscillations with a higher frequency coherence. Time-resolved LIF studies have been performed on Hall thrusters of different power classes, from a few hundred ${ }^{19-21}$ to a few thousand ${ }^{22,23}$ Watts, revealing temporal modulations in the ion velocity and probed metastable ion density due to the breathing mode; however, many of these studies have been limited to the investigation of few locations in the plasma, both discharge channel and plume, due to the onerous resources (time, equipment, computational or data processing) needed for such challenging measurements.

The present study applies a time-resolved continuouswave LIF technique known as the sample-hold method to measure dynamic velocity distributions in an unprecedented number of spatial locations in the plume of a commercial BHT-600 Hall thruster operating in a strong, natural, breathing mode condition. Xenon atoms undergo ionization and subsequent acceleration in the thruster channel, as detailed in a previous publication, ${ }^{15}$ before entering the complex and dynamic environment of the thruster plume. In this study, ion velocity distributions obtained throughout the near-field and far-field regions, including the central jet, are resolved in two spatial dimensions over the oscillation period. Measurements track the dominant high velocity ion populations from the channel in addition to secondary populations with much lower velocity. This work contributes a rich time-resolved dataset for model validation and calibration, and lends new insight into the complex dynamics of the thruster plume region under action of a strong breathing mode.

The paper is organized as follows: section II briefly describes the time-resolved LIF approach and details the experimental facility, BHT-600 thruster under study, and LIF measurement configuration. Section III discusses the time-resolved LIF results characterizing first the broader near-field dynamic, followed by the structure of the central jet. Some conclusions are offered in Section IV.

\section{EXPERIMENTAL METHODS}

\section{A. BHT-600 Hall Thruster Test Setup}

The plasma propulsion system investigated in this study is a commercial BHT-600 Hall Effect Thruster ${ }^{24,25}$ coupled with a BHC-1500 barium oxide hollow cathode (Fig. 1), both operated on xenon with mass flow rates of $2.21 \mathrm{mg} / \mathrm{s}$ and $0.15 \mathrm{mg} / \mathrm{s}$, respectively. The discharge channel features an outer radius of $32 \mathrm{~mm}$, an inner radius of $24 \mathrm{~mm}$ and a depth of $10 \mathrm{~mm}$. The thruster is operated at an anode power of about $600 \mathrm{~W}$, with an anode voltage of $300 \mathrm{~V}$ and a discharge current of 2.05$2.15 \mathrm{~A}$. The magnetic field is generated by the magnetic coils of the thruster, operated with a current of 1.75 A. Under these operating conditions, the BHT-600 experiences strong breathing mode plasma oscillations with the fundamental harmonic in the $43-50 \mathrm{kHz}$ range. Representative traces of the discharge current, discharge voltage and optical emission signal (captured through a monochromator-PMT assembly monitoring the $542 \mathrm{~nm}$ ion line) are shown in Fig. 2 along with their spectral contents computed through an FFT. All three spectra show a well-defined fundamental feature at $48 \mathrm{kHz}$ as well as higher harmonics.

The experimental measurements are performed in the $1.8 \mathrm{~m}$ diameter, $3 \mathrm{~m}$ long Chamber 6 at the Air Force Research Laboratory (AFRL) at Edwards Air Force Base, $\mathrm{CA}$. The chamber is equipped with four single stage cryogenic panels and a two-stage cryogenic pump providing an overall pumping capacity of $32000 \mathrm{~L} / \mathrm{s}$ for xenon, achieving a base pressure of about $1 \times 10^{-6}$ Torr and a pressure of $4 \times 10^{-5}$ Torr during thruster operation. The thruster is mounted on a three-axis motorized stage assembly with sub-millimeter resolution.

\section{B. Time-Resolved Laser-Induced Fluorescence}

The ion velocity distribution function in the BHT-600 plume is mapped in both time and space domains during breathing mode oscillations using the sample-hold time resolution architecture conceived at the Stanford Plasma Physics Laboratory and extensively described in previous publications. ${ }^{15,26}$ In the AFRL experimental setup, a New Focus TA-7600 tapered amplifier seeded by a TLB6817 tunable diode laser produces $\sim 23 \mathrm{~mW}$ of diode laser power to optically pump the $5 d[4]_{7 / 2}-6 p[3]_{5 / 2}^{\mathrm{o}}$ Xe II transition $(834.953 \mathrm{~nm}$ vacuum). Using beam samplers and mirrors, portions of the laser beam are diverted into an optogalvanic cell and a Fabry-Pérot (F-P) etalon for an accurate determination of the laser wavelength during the scan. The $6 p^{\prime}[3 / 2]_{1}-8 s^{\prime}[3 / 2]_{1}$ Xe I transition $(834.974 \mathrm{~nm}$ vacuum) in the optogalvanic cell, spaced $\sim 10 \mathrm{GHz}$ from the target stationary ion transition, is used for the zero velocity reference, while the F-P etalon provides fixed frequency markers every $\mathrm{FSR}=0.3 \mathrm{GHz}$.

Two laser beams, oriented perpendicular to each other, are used to simultaneously measure two ion velocity components: one beam is parallel to the thruster axis for axial velocity measurements $(-\hat{z}$ in Fig. 1$)$, and a second beam runs parallel to the exit plane of the thruster for radial velocity measurements $(+\hat{x}$ direction). The resulting fluorescence emitted at $542.1 \mathrm{~nm}$ (vacuum) from the radiative relaxation to the $6 s[2]_{3 / 2}$ state is collected by two $100 \mathrm{~mm}$ diameter, $200 \mathrm{~mm}$ focal length lenses with a collection axis at $\sim 60^{\circ}$ with respect to the thruster axis. The fluorescence is filtered through a monochromator with a bandwidth of a few nanometers, and detected by an Hamamatsu R928 PMT for determining the Doppler 


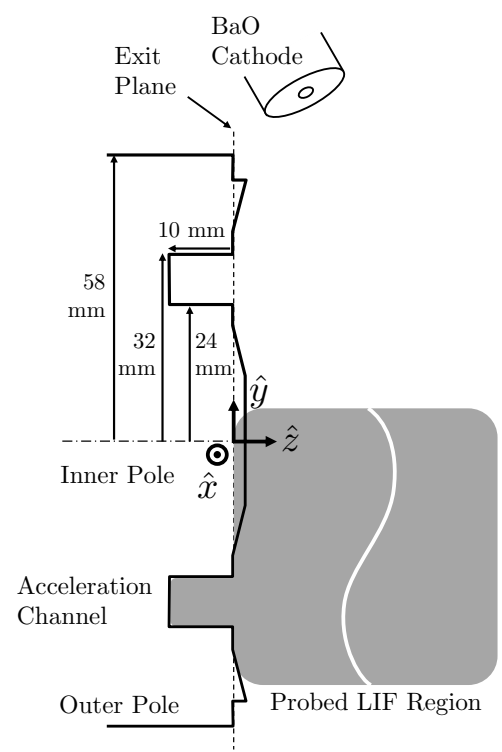

(a)

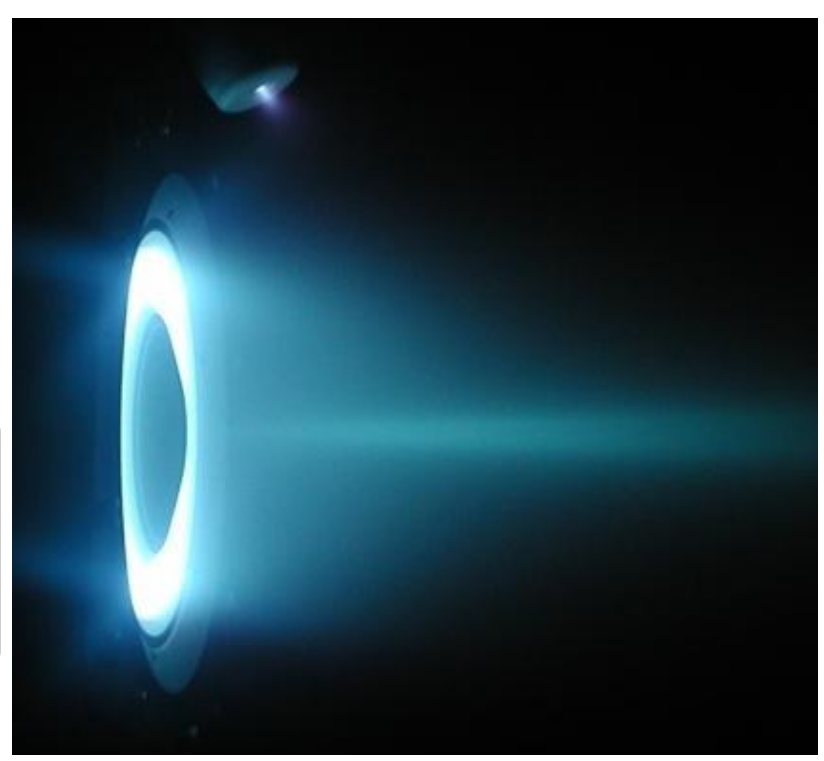

(b)

FIG. 1: (a) Diagram of BHT-600 Hall thruster, including thruster dimensions and the coordinate system used in this work. (b) Side view of BHT-600 Hall thruster operating on xenon. The external cathode is visible above the thruster. Reproduced with permission from Plasma Sources Sci. Technol. 27, 094004 (2018). Copyright 2018 IOP Publishing.

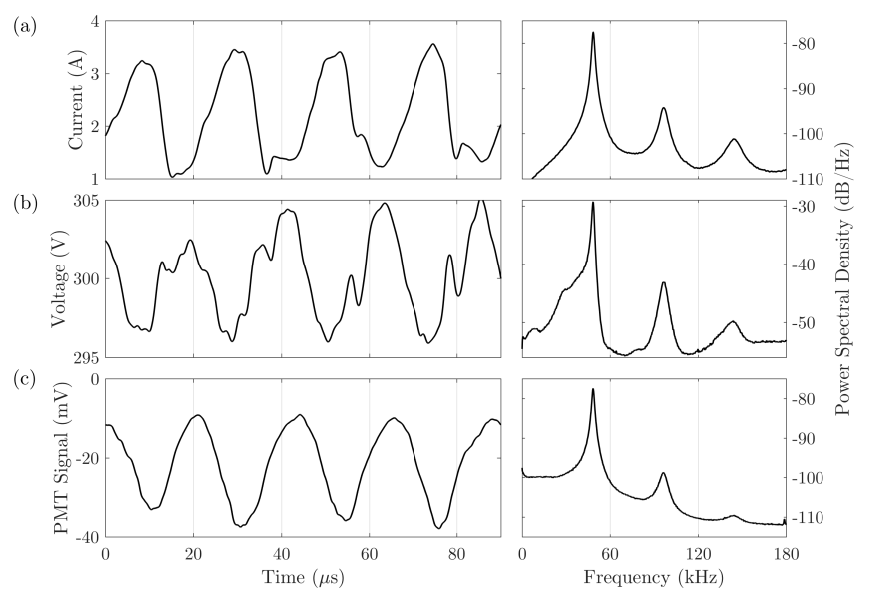

FIG. 2: Left column: (a) discharge current, (b) discharge voltage, and (c) plasma emission signals captured from the BHT-600. Right column: spectral content of each signal showing the dominant breathing mode at $48 \mathrm{kHz}$. Note that the photomultiplier tube (PMT) signal is negative, indicating increasing plasma emission in the downward direction. Reproduced with permission from Plasma Sources Sci. Technol. 27,

094004 (2018). Copyright 2018 IOP Publishing.

shift of the fluorescence excitation spectrum with respect to the stationary reference at a given point in the thruster plume. Note that the LIF signal intensity is proportional to the metastable ion density, while numerical analy- ses usually report the global ion density. A complete collisional-radiative model, ${ }^{27,28}$ including higher excited states and detailed electron dynamics, would be required in order to give a complete picture of the excited state populations and, in particular, fully resolve the relationship between ground and metastable ion densities.

The PMT signal is processed according to the samplehold scheme, as illustrated in Fig. 3. The signal originating during a short time window (synchronized to a certain phase of discharge current) is sampled, averaged and held by a dedicated circuit until the next acquisition gate one discharge current cycle later, at which point the signal level is updated. The acquisition gates of a certain width are generated by a digital delay generator with a given delay from a zero phase trigger. The latter is produced by a voltage comparator when the discharge current passes through a tunable threshold, indicating the start of a new cycle. The sample-hold scheme excludes the signal originating outside the desired phase in the current cycle and passes the signal sampled and averaged in the acquisition gate to a lock-in amplifier for homodyne detection. While the acquisition gate is parked at a certain phase of the oscillation period (i.e. at a certain delay from the trigger), a full laser wavelength scan is performed, providing a measurement of the LIF transition lineshape at that specific phase in the current oscillation cycle. The method achieves high resolution in laser wavelength/velocity space and must be repeated at various delays along the full current cycle to reconstruct the temporal evolution of the LIF lineshape.

Nine sample-hold branches, each with their own dedi- 
cated sample-hold circuit and lock-in amplifier, are used in parallel to simultaneously extract LIF lineshapes at several phase delays. A tenth channel provides a dedicated time-averaged LIF trace. With three laser wavelength scans per measurement location, twenty-seven phase points within the $20 \mathrm{~s}$ oscillation period are taken at $1 \mathrm{~s}$ increments with a gate width of $1 \mathrm{~s}$, with additional resolution obtained at half microsecond intervals between $3-9 \mathrm{~s}$.

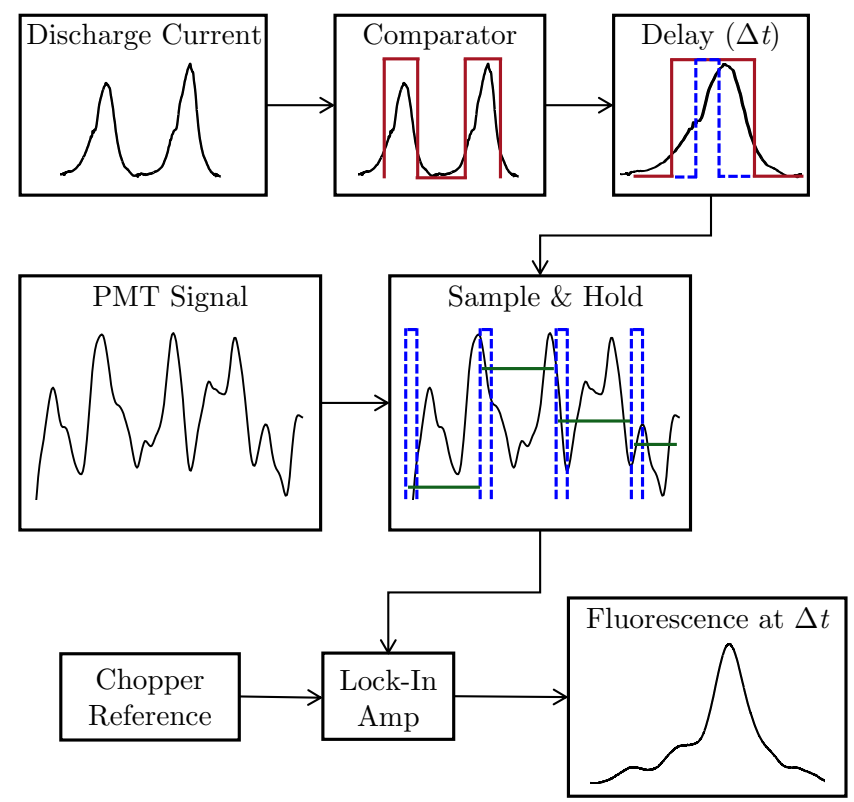

FIG. 3: Block diagram of the sample-hold method for time-resolved laser-induced fluorescence spectroscopy. A comparator chip triggers the creation of a measurement gate of width $\tau$ and delay time $\Delta t$ from the point at which the discharge current crosses a defined threshold The fluorescence signal from the PMT is sampled, held until the following update one discharge current cycle later, and fed into a lock-in amplifier that extracts the LIF lineshape at the chosen phase in the current cycle. Reproduced with permission from Plasma Sources Sci.

Technol. 27, 094004 (2018). Copyright 2018 IOP Publishing.

\section{RESULTS AND DISCUSSION}

\section{A. Plume}

Time-resolved LIF measurements are obtained at multiple spatial locations, providing fundamental knowledge on the space- and time-varying ion velocity distribution function. The reference frame used in this analysis is shown in Fig. 1. Note that the cathode is located above the channel in the $+\hat{y}$ direction, and the collection lens is located on the $-\hat{x}$ side of the thruster. The axiallyoriented exciting laser is launched in the $-\hat{z}$ direction,

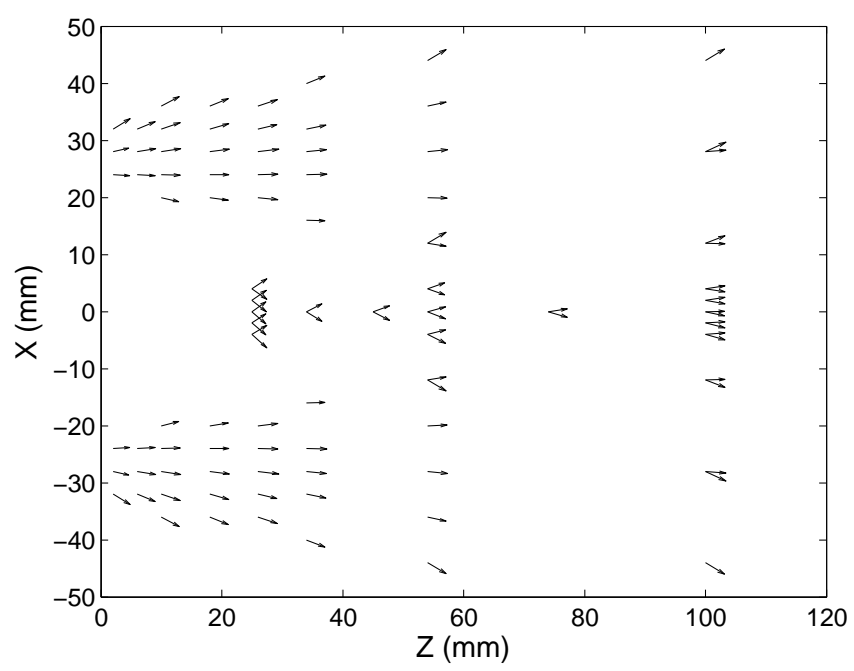

FIG. 4: BHT-600 time-averaged 2-D ion velocity vectors obtained in the $X Z$ plane. Compare with Fig. 5 from Ref. 29. Note that data for $x>10 \mathrm{~mm}$ has been reflected from below for visual effect.

and the resulting Doppler shifts are plotted such that ions with a positive velocity are traveling in the $+\hat{z}$ direction.

Figure 4 shows the time-averaged velocity vectors calculated from the most probable axial and radial velocities obtained at each point in separate LIF measurements. Note that data appearing for $x>10 \mathrm{~mm}$ has been reflected across the axis for visual effect in Fig. 4. Near the thruster axis, multiple dominant radial populations are visible due to the crossing of the ion beams from opposite sides of the channel (more on this in Section IIIB). Not represented in this plot are secondary axial populations of slower ions that arise at certain locations near the exit plane and central jet (see Section III C). This timeaveraged picture of the BHT-600 plume closely matches previous time-averaged LIF studies of this thruster. ${ }^{29}$

The observed modulations in the axial and radial velocities over time are too small relative to their average values to produce noticeable differences in the magnitude and direction of the velocity vectors visualized in this way. Alternate viewpoints are required to examine 2-D ion dynamics in the plume. We introduce the following normalization as a convenient way to compare measurements of a modulating quantity $x(t)$ that might vary substantially in mean value or fluctuation amplitude across the dataset, while maintaining relative phase information: $\left(x(t)-x_{\min }\right) /\left(x_{\max }-x_{\min }\right)$. (Note that for velocities, $v_{\max }$ and $v_{\min }$ are taken in the direction of travel.) For every LIF measurement location, this normalization is applied to the observed time histories of the most probable ion velocity and peak LIF intensity.

The relative phase information in the time-resolved measurements (i.e. the relative point in the discharge current oscillation at which the ion velocity or peak intensity reach their maximum or minimum values) taken at dif- 
ferent spatial locations reveals interesting features about the plume dynamics. Cross-correlation is used to identify a characteristic phase lag (in s) between each modulating quantity and the (similarly normalized) BHT-600 discharge current trace. The cross-correlation of two discrete signals $x$ and $y$ is given by:

$$
r_{x y}[l]=\sum_{n=1}^{N} x[n] y[n-l]
$$

where $r_{x y}$ is the cross-correlation coefficient between the signals for time lag $l$. The index $n$ is taken at $1 \mathrm{~s}$ intervals between 0 and $20 \mathrm{~s}$ over the breathing mode period $(N=21)$. The value of $l$ that maximizes the crosscorrelation coefficient is the amount by which $y$ lags the signal $x$ in time. Here, $x$ is taken to be the discharge current and $y$ is the normalized velocity or intensity. The reported lag values are periodic, and negative results (phase lead) are simply rescaled to positive values by adding one oscillation period. A lag value of 0 or $20 \mathrm{~s}$ indicates the modulating quantity is in phase with the current, while a lag value of $10-11$ s indicates a $180^{\circ}$ phase shift. In this way the modulations throughout the plume may be compared.

Before moving to summary plots for the whole plume, it is constructive to examine the raw signal traces at a few points. Fig. 5 summarizes the trends in the both the axial $(\mathrm{a}, \mathrm{c})$ and radial $(\mathrm{b}, \mathrm{d})$ LIF signals as one moves downstream straight out along the channel midline, $x=-28 \mathrm{~mm}(\mathrm{a}, \mathrm{b})$, and diagonally towards the main thruster axis, $x=0$ (c,d). In each panel, the normalized most probable velocity appears at the top, followed by normalized peak intensity, and discharge current at the bottom. The small boxes to the right of each plot indicate the time-averaged quantity normalized in a similar way. The cross-correlation returns a single representative time delay value for each trace. Table I summarizes the cross-correlation results for the data of Fig. 5. Note that for very small modulations on top of a large mean velocity (small $\delta v / v$ ) the trends become noisier due to uncertainty in the peak fitting. LIF traces taken further out in the plume also tend to have lower signal to noise ratio due to fewer ions in the measurement volume.

\begin{tabular}{|c|c|c|c|c|c|c|}
\hline & & & Del & ay (s) & & \\
\hline$x(\mathrm{~mm})$ & $z(\mathrm{~mm})$ & Ax. Vel. & Ax. Int. & Rad. Vel. & Rad. Int. & Fig. 5 \\
\hline-28 & 6 & 14 & 2 & 0 & 3 & $(\mathrm{a}, \mathrm{b}, \mathrm{c}, \mathrm{d})$ \\
\hline-28 & 54 & 4 & 5 & 9 & 9 & $(\mathrm{a}, \mathrm{b})$ \\
\hline-28 & 100 & 1 & 5 & 3 & 9 & (a,b) \\
\hline-16 & 34 & 19 & 5 & 15 & 5 & $(\mathrm{c}, \mathrm{d})$ \\
\hline 0 & 74 & 18 & 1 & 0 & 9 & $(\mathrm{c}, \mathrm{d})$ \\
\hline
\end{tabular}

TABLE I: Characteristic delay times between measured LIF data (ion velocity and LIF intensity modulation waveforms for both radial and axial LIF) and the

breathing mode discharge current trace calculated via cross-correlation. The corresponding LIF waveforms appear in the indicated panels of Fig. 5.

Comparing the normalized velocity and intensity modulations in this way illuminates some trends in the data.
For example, in Fig. 5a the time delay between axial velocity modulations and the current increases by about half a period between the exit plane and the far-field plume, while the intensity modulations are fairly consistent. The radial velocity modulations in Fig. 5b also show a clear shift, with lag time increasing between the exit plane and the far plume. Here the intensities downstream reach their peaks around $6 \mathrm{~s}$ after the point at $z=6 \mathrm{~mm}$. When moving towards the main thruster axis in Figs. $5 \mathrm{c}-\mathrm{d}$, the axial velocities show a similar increase in delay time moving downstream, while the axial intensities are offset from each other by a few microseconds. The radial velocity and intensity modulations track the current more closely in this direction.

By computing a cross-correlation for each of the four measured quantities at every LIF point, spatially resolved maps of the velocity and intensity fluctuations throughout the plume may be constructed. These are displayed in Fig. 6. Due to noise in the LIF traces that propagates through the Gaussian peak fitting procedure into the modulation waveforms (see Fig. 5), these maps should be viewed with caution in a qualitative capacity only. However, they are useful for generally tracking how the dynamics of different areas of the plume are progressing relative to one another. Most probable velocity modulations are presented in (a) for axial LIF and (b) for radial LIF, and LIF peak intensity modulations appear in (c) for axial LIF and (d) for radial LIF. Both the normalized modulation waveforms and the resulting 2-D color maps have been lightly smoothed ${ }^{30}$ and the weighted interpolation scheme discussed in the prior time-resolved LIF studies of the BHT-600 channel ${ }^{15}$ has been applied.

The plots of Fig. 6 confirm the trends suggested by the few points examined in Fig. 5. In general, the lag time between the discharge current and the measured quantity increases with distance away from the channel (located outside the plotting area at: $-32<x<-24$, $z \leq 0)$. The largest gradient observed for axial velocity is in the $\hat{z}$ direction, and the largest gradient in radial velocity is in the $\hat{x}$ direction, as one might expect. The intensity modulations are close to in phase with the discharge current near the channel exit, as expected from our previous measurements within the channel. ${ }^{15}$ The increasing delay time with distance is a consequence of the finite ion transit time between the channel and the measurement point in the plume. For example, a burst of ions leaving the exit plane in phase with the current will cause the LIF signal to be maximized at progressively later times the farther the ions travel downstream. At $15 \mathrm{~km} / \mathrm{s}$, an ion will traverse $1 \mathrm{~cm}$ in $6.7 \mathrm{~s}$, on the order of the increase in lag times observed here. This situation was exploited by Bouchoule et al. ${ }^{11}$ to reconstruct the breathing mode dynamics at the exit plane of an SPT100 Hall thruster using a retarding field energy analyzer placed $80 \mathrm{~cm}$ downstream in the plume and correcting for the ion time-of-flight to reach the detector.

This effect is better illustrated by plotting the actual normalized modulation waveforms as a function of time 


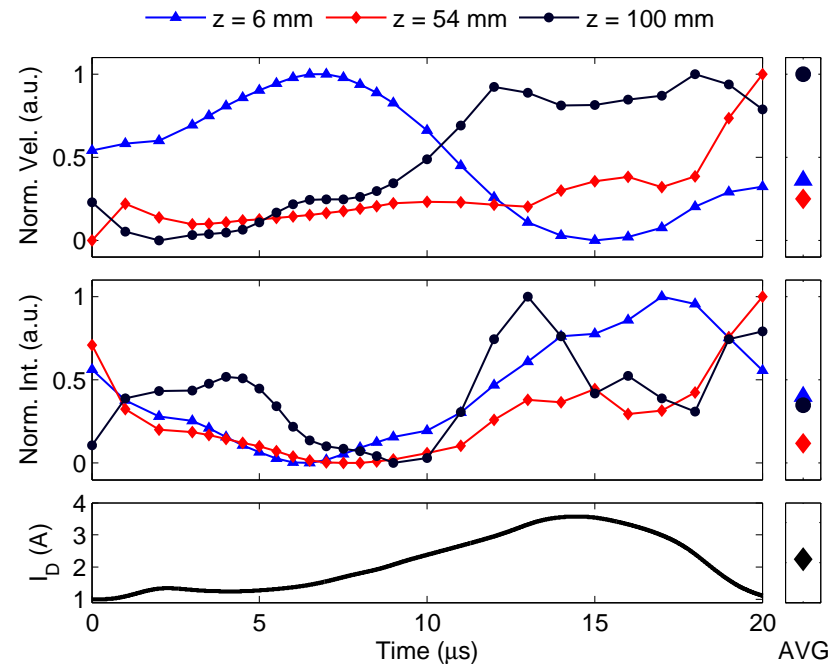

(a) $x=-28 \mathrm{~mm}$, Axial LIF

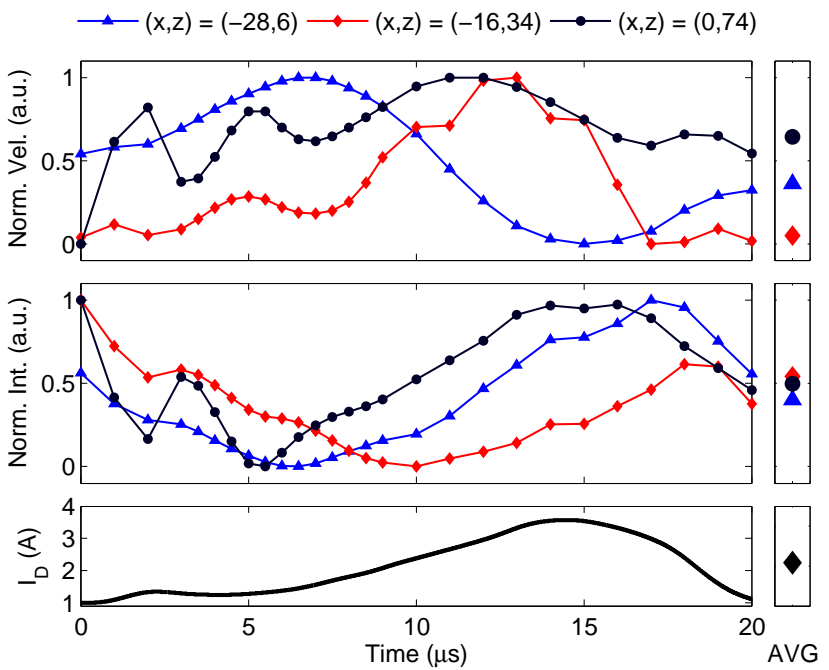

(c) Diagonal, Axial LIF
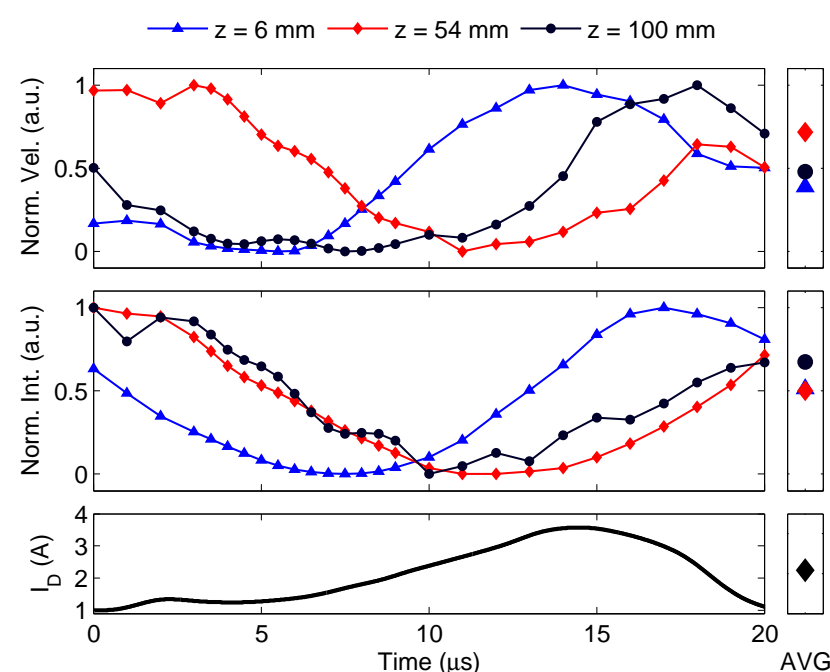

(b) $x=-28 \mathrm{~mm}$, Radial LIF

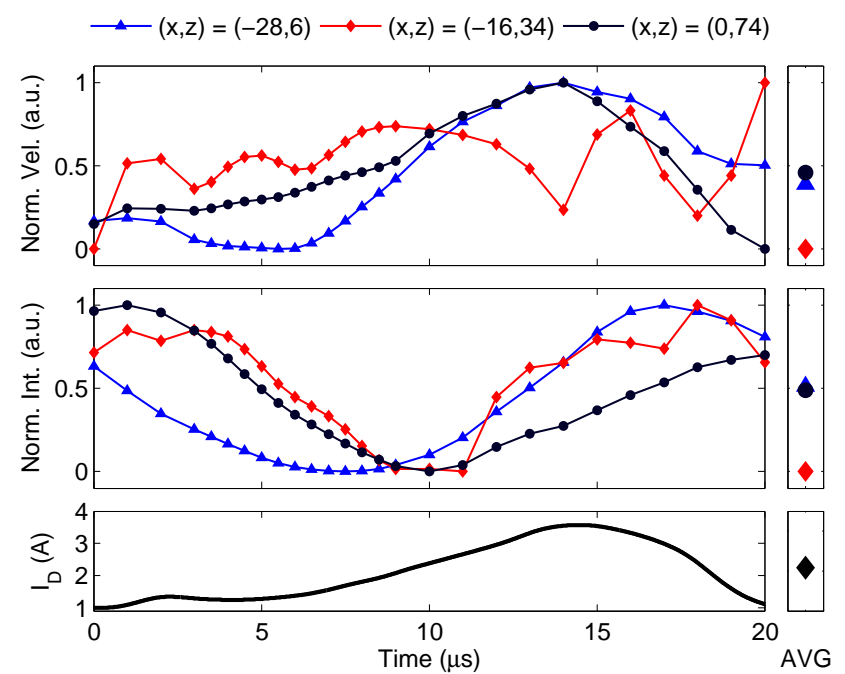

(d) Diagonal, Radial LIF

FIG. 5: Normalized axial (a,c) and radial (b,d) ion velocity and LIF intensity modulations at a few points in the BHT-600 plume showing an increase in signal delay times with distance from the channel. (a,b) Variation along a line moving axially downstream along the channel centerline. (c,d) Variation along a line moving diagonally inward towards the main thruster axis. The boxes to the right of each panel show the corresponding time-averaged measurement (AVG).

throughout the domain. The columns of Fig. 7 show the relative oscillation phase of the measured quantities at all points in the plume at different snapshots in time. Again, the images are smoothed and interpolated. The columns in order from left to right detail the normalized axial velocity, radial velocity, axial peak intensity, and radial peak intensity. The time points correspond to the discharge current traces of Fig. 5.

The downstream effects of the strong breathing mode oscillations originating in the discharge channel become apparent and the observed trends seen here can be linked to the behavior seen in our prior work ${ }^{15}$ and in other ex- perimental observations of Hall Thruster plume dynamics. ${ }^{14}$ In the BHT-600 channel during the time interval between $20 \mathrm{~s}$ and $2 \mathrm{~s}$ after the trigger, the electric field peaks and has traveled farthest upstream. At this point ions experience the greatest acceleration inside the channel, attaining their near maximum velocity in the area immediately beyond the exit plane a few microseconds later, between $t=0-8 \mathrm{~s}$. The LIF intensity is also lowest during this interval in the discharge current trough as the ions accelerate. As the ionization and acceleration zones move back upstream to start the next cycle, the ion velocity diminishes at the channel exit. We can now track the 


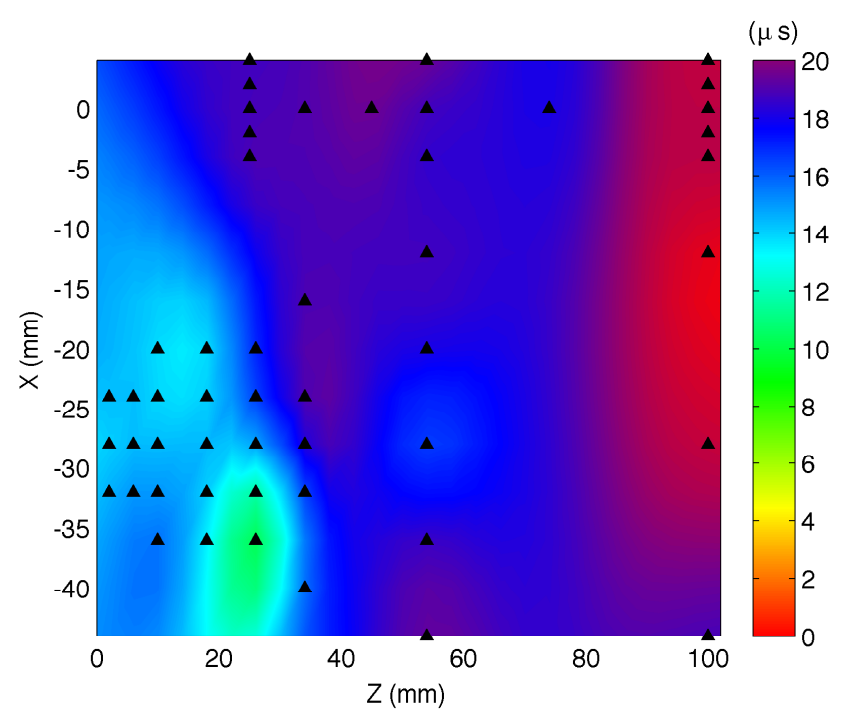

(a) Axial Velocity

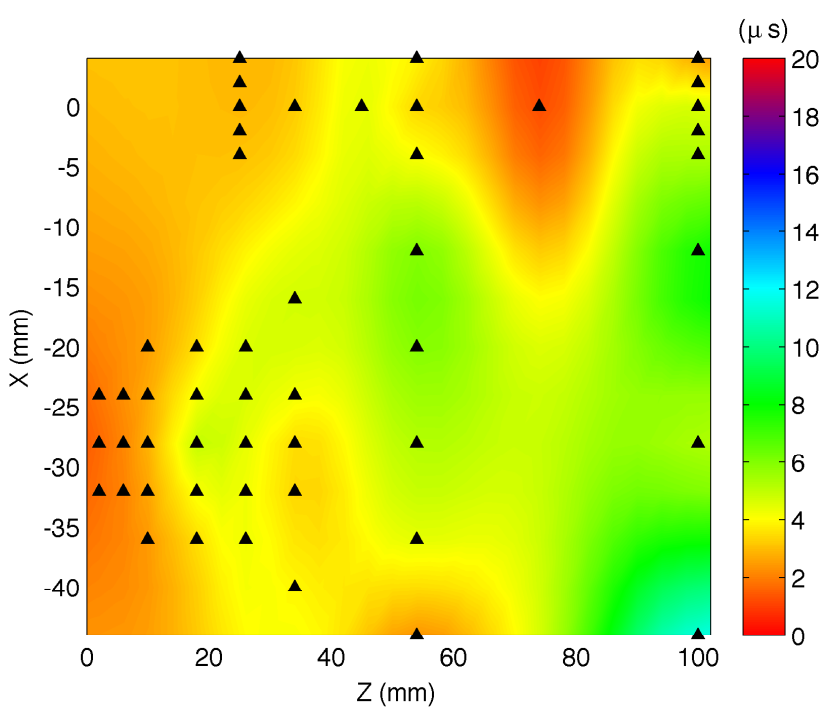

(c) Axial Intensity

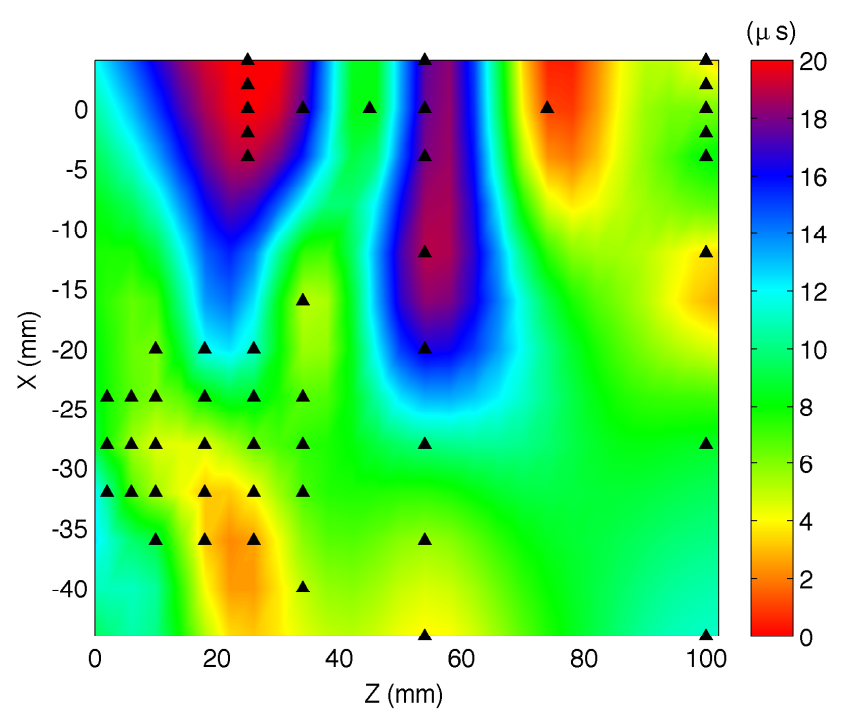

(b) Radial Velocity

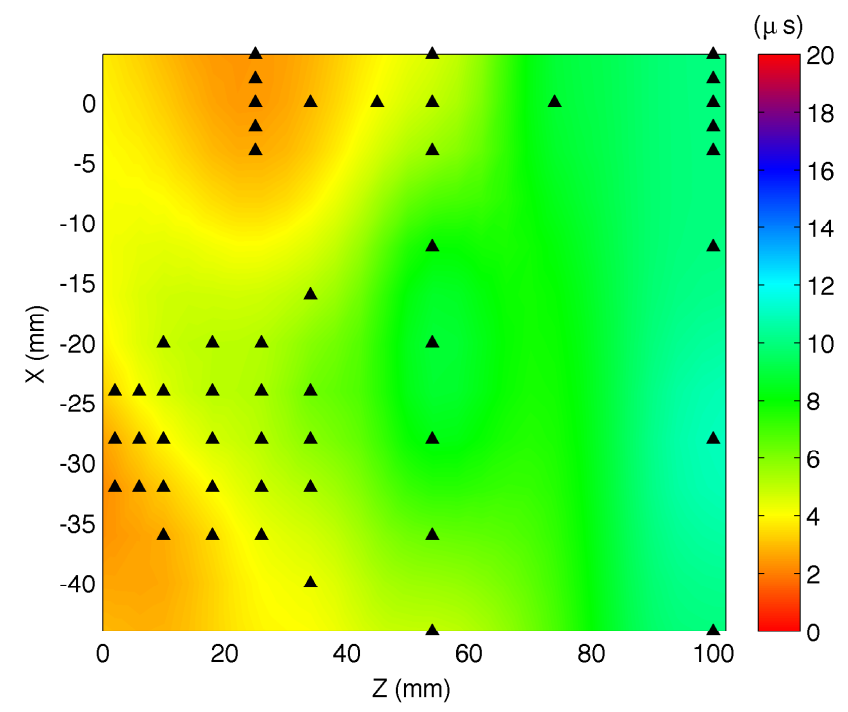

(d) Radial Intensity

FIG. 6: Maps of most probable velocity (a,b) and peak LIF intensity $(\mathrm{c}, \mathrm{d})$ modulations throughout the BHT-600 plume derived from axial $(\mathrm{a}, \mathrm{c})$ and radial $(\mathrm{b}, \mathrm{d})$ LIF measurements. Each waveform undergoes a cross-correlation with the discharge current, producing a representative delay time. Note that the time scale, and thus color scales, are periodic.

general ion trajectories into the plume as both axial and radial velocity components reach their peak modulation during the following interval between 11-15 s.

LIF intensities remain low throughout the plume until around $15 \mathrm{~s}$, when the ions begin to slow. Then, as the ions near the channel attain their lowest velocities while the current ramps up (15-19s), both the radial and axial LIF intensity builds into the near-field plume. Farther downstream at $z=100 \mathrm{~mm}$, however, the intensity modulation is essentially $180^{\circ}$ out of phase with the near field, and an intermediate value is attained at the halfway points of $z=54 \mathrm{~mm}$. The radial intensity data especially illustrates this effect of a propagating "wave" in which points successively farther from the discharge channel reach their peak intensity (and assumed peak ion density) at later and later times. At $4 \mathrm{~s}$, as the next high velocity burst of ions is about to exit the channel, the previous cycle of ions is still visible at the $z=100 \mathrm{~mm}$ points. These data show how the phase difference between the intensity and velocity modulations initially established by the breathing mode dynamics in the channel continues out into the plume, where a strong spatial and temporal dependence in the modulations of the measured quantities is apparent. 

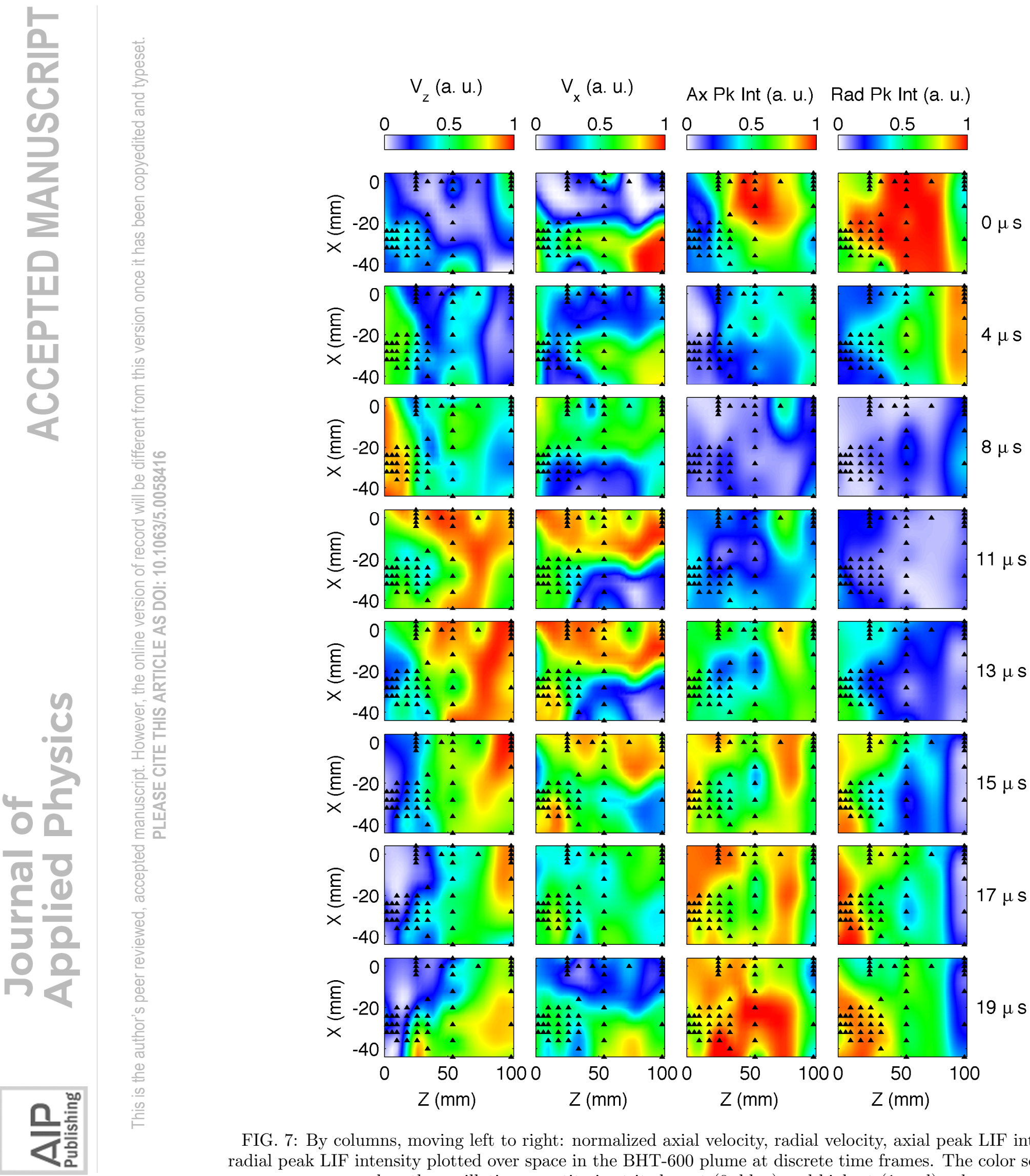

FIG. 7: By columns, moving left to right: normalized axial velocity, radial velocity, axial peak LIF intensity, and radial peak LIF intensity plotted over space in the BHT-600 plume at discrete time frames. The color scale indicates when the oscillating quantity is at its lowest $(0$, blue) and highest $(1$, red) values. 


\section{B. Central Jet}

The central jet of the BHT-600 is visible in the photo of Fig. 1, emanating from a point near the inner pole ceramic. The precise origin and nature of this conical region of high visible emission in Hall thrusters is still not fully understood and has been the subject of time-averaged laser-induced fluorescence, ${ }^{29}$ probebased, ${ }^{31}$ and theoretical ${ }^{32}$ investigations, as well as electron trajectory analyses. ${ }^{33,34}$ The appearance of the jet is correlated with high efficiency thruster performance, and is sensitive to operating voltage and facility back pressure. It likely provides a high-conductivity path for electrons that reduces voltage losses in the plume, and it may contribute to external ionization of neutrals. It is clearly a region of flow mixing between the diverging beams emanating from the annular channel that meet along the main axis. Possible explanations for its presence include ion collisional excitation and an ion acoustic shock (its conical structure is reminiscent of an oblique shock in a supersonic neutral flow). ${ }^{32}$

The center jet region is interrogated at several points, including several near the center pole piece, and also farther into the plume. The time-averaged traces (dashed lines) and integral average of the time-resolved traces (solid lines) are shown in Figs. $8 \mathrm{a}$ and 8b, their close agreement providing a sanity check of the time-resolved sample-hold LIF method. The corresponding 2-D vectors derived from the most probable velocities appear in Fig. 8c. Note that the plots are oriented so the plume is pointing towards the top of the page. In Fig. 8a, each LIF trace has been normalized to its maximum intensity value to facilitate comparison. The traces are stacked vertically according to axial $(\hat{z})$ location.

Beginning with the time-averaged axial traces in the top row of Fig. 8a, the data show some ion acceleration is occurring within the central jet between 25 and $100 \mathrm{~mm}$ downstream. When comparing the off-axis traces at $x= \pm 2 \mathrm{~mm}$ and $x= \pm 4 \mathrm{~mm}$, no significant left-right asymmetry is observed in the axial data. Moving to the radial data in the bottom row of Fig. 8a, one observes multiple dominant radial populations as the ions originating from opposite sides of the discharge channel cross within the LIF measurement volume. Additionally, there is significant signal between peaks indicating populations of low velocity ions. The radial peaks along $x=0$ move closer together with increasing axial distance from the thruster, indicating a reduction in the radial component of the velocity vectors. This is consistent with a geometric view of the ion flows crossing on axis, in which only ions with increasingly slower radial velocity components will reach the measurement volume when moving downstream.

The relative intensities of the two peaks in the radial data give a clue as to which beam of ions (i.e. those originating from the $+\hat{x}$ or $-\hat{x}$ side of the channel) is dominant in that location. Geometrically, ions emitted towards the main thruster axis from the channel at $x=-28 \mathrm{~mm}$ will have a positively directed velocity, and ions emitted from the channel at $x=+28 \mathrm{~mm}$ will have a negatively directed velocity. Along the line of measurement points at $x=-4 \mathrm{~mm}$, for example, the taller radial LIF peaks appear on the side of positive velocity, as we have moved in the direction of the $-\hat{x}$ channel. The negative velocity peaks, originating from the opposite side of the channel farther away, are correspondingly smaller.

An asymmetry is clearly apparent in the radial data. In the time-averaged traces, the beam of positive radial velocity from the $-\hat{x}$ side of the channel is dominant until $z=100 \mathrm{~mm}$ downstream. In fact, at $z=25 \mathrm{~mm}$, one has to move $4 \mathrm{~mm}$ in the $+\hat{x}$ direction until the radial LIF signals from each side are of equal weight. The magnitude of this shift precludes alignment error as the cause of the asymmetry, as the spatial uncertainty in the coordinate system zero is estimated to be $\sim 1 \mathrm{~mm}$ at most, and the motorized translation stages are accurate to hundreds of microns. A similar asymmetry was revealed in a previous time-averaged LIF characterization of this thruster, ${ }^{35}$ although measurements were made in the $Y Z$ plane with the cathode located at the $-\hat{y}$ channel position. In that study, the point at which opposing radial LIF peaks showed equal weight was shifted $1-2 \mathrm{~mm}$ in the $+\hat{y}$ direction, away from the cathode. In the $X Z$ plane of the present study, however, the cathode located at the $+\hat{y}$ position should affect both sides equally. This suggests that additional mechanisms besides the cathode asymmetry are at work in the central jet region. Possible explanations include asymmetric neutral injection due to manufacturing defects or wear, and nonuniform erosion of the wall, anode, or front face plate. Further investigation is required to provide a conclusive assessment.

As seen from the centerline data along $x=0$, there is also an asymmetry in the widths of the radial distributions originating from each side, accentuated as ions move farther downstream. It appears that a narrower radial population of ions is generated from the $+\hat{x}$ side of the channel, while the positive velocity half of the IVDFs from the $-\hat{x}$ side have greater width. Such a broadening of the time-averaged IVDF could be the result of a dynamic effect like that illustrated in a previous study of a lower power Hall thruster (Z-70), ${ }^{21}$ or a physical ionization process that creates and accelerates many ions to differing velocities. A look at the time-resolved LIF traces in this area in Fig. 9 does not show the same sort of large oscillations in the LIF peak location seen previously in the Z-70, however; the distributions maintain their width roughly for all time. If the central jet is indeed a pathway for significant external ionization of neutrals as suggested by Hruby et al., ${ }^{32}$ these "slow" ions could eventually make their way downstream and add to the perceived width of the radial populations derived from the main channel. Whatever physics is behind the observed asymmetry would push these slower ions preferentially in the $+\hat{x}$ direction to be recovered here as a broader LIF trace. Examining the multiple axial populations visible in the time-resolved data for $z<25 \mathrm{~mm}$ 

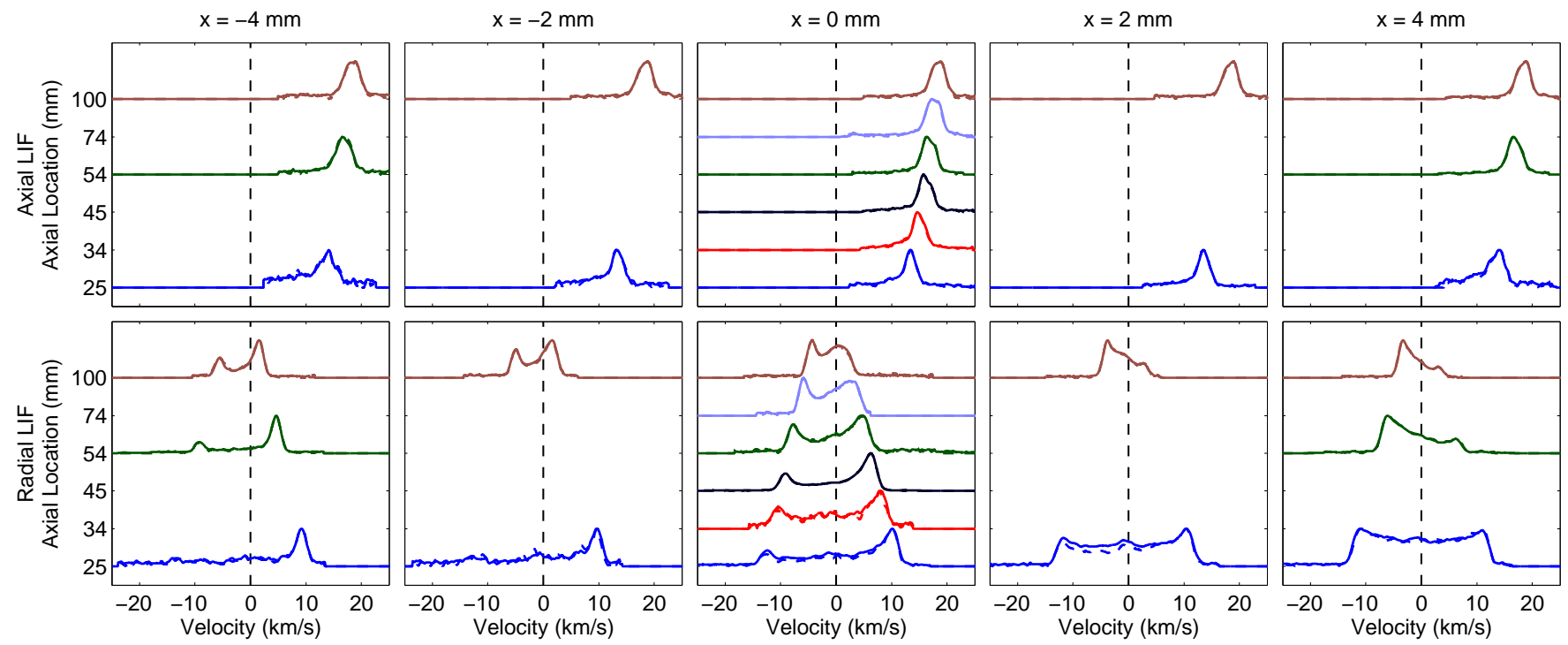

(a)

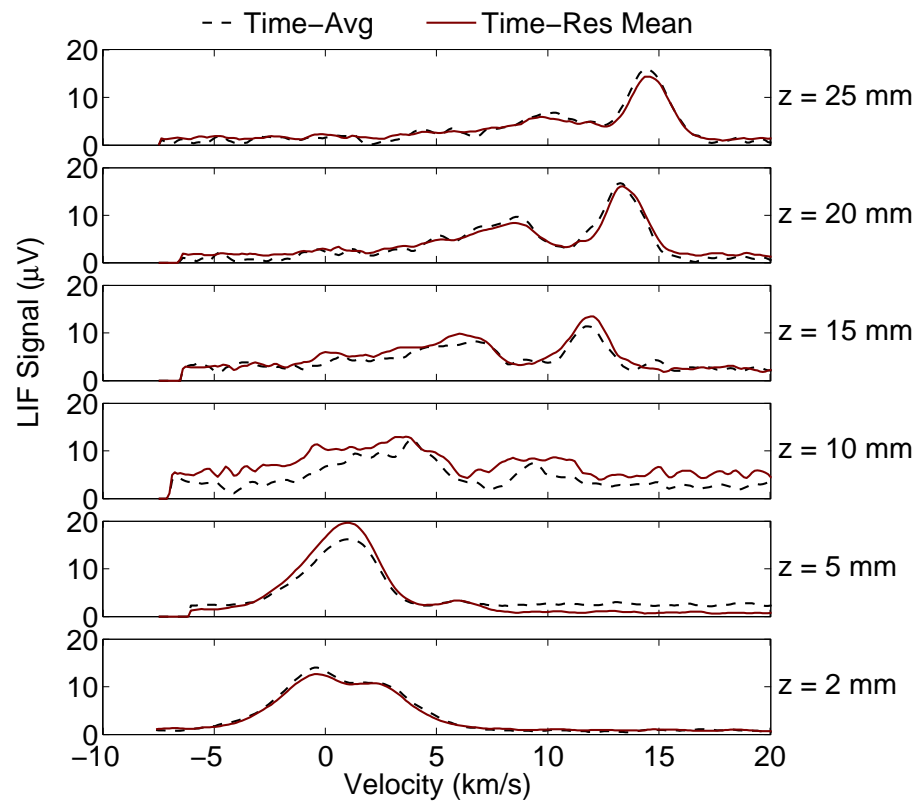

(b)

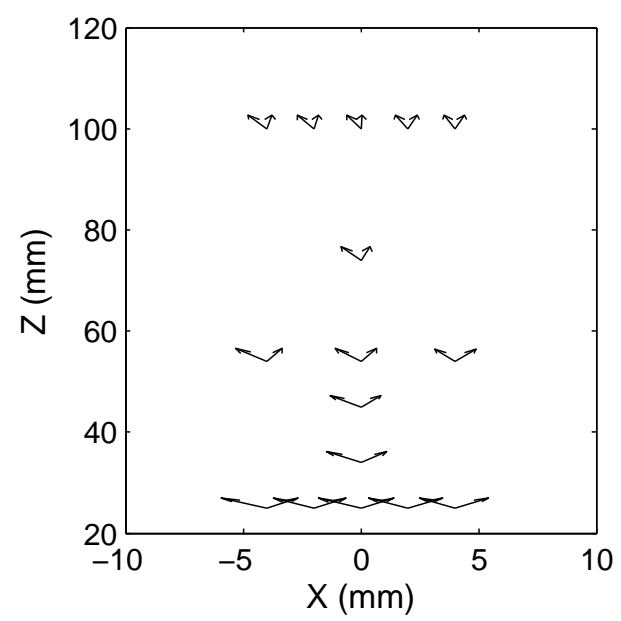

(c)

FIG. 8: (a) Time-averaged axial (top row) and radial (bottom row) LIF traces obtained at various points in the center jet region of the BHT-600. (b) Time-averaged axial LIF traces obtained closer to the thruster. Dotted lines indicate time-averaged traces, while the solid lines show the integral average of the time-resolved data to verify agreement. (c) Velocity vectors derived from the most-probable 2-D velocity components in (a), for reference.

(see Fig. 11) lends support to this theory.

Before moving closer to the thruster, one additional interesting dynamic effect is observed in the axial ion populations down the thruster axis, $x=0$. As seen in Fig. 10, the axial component of the two ion beams originating from each side of the channel is very similar. This is expected, since ions from each side have traveled essentially the same distance to the measurement point.
However, at different times during the breathing mode current cycle, the axial LIF peak is seen to shift from one side to the other, with transitions occurring at 8-9s and $17 \mathrm{~s}$. This suggests that for about half the discharge cycle, more ions are arriving from one side of the channel to slightly dominate the LIF peak. About halfway up the current ramp, and halfway down the other side, the dominant population switches. During the $8-17 \mathrm{~s}$ in- 


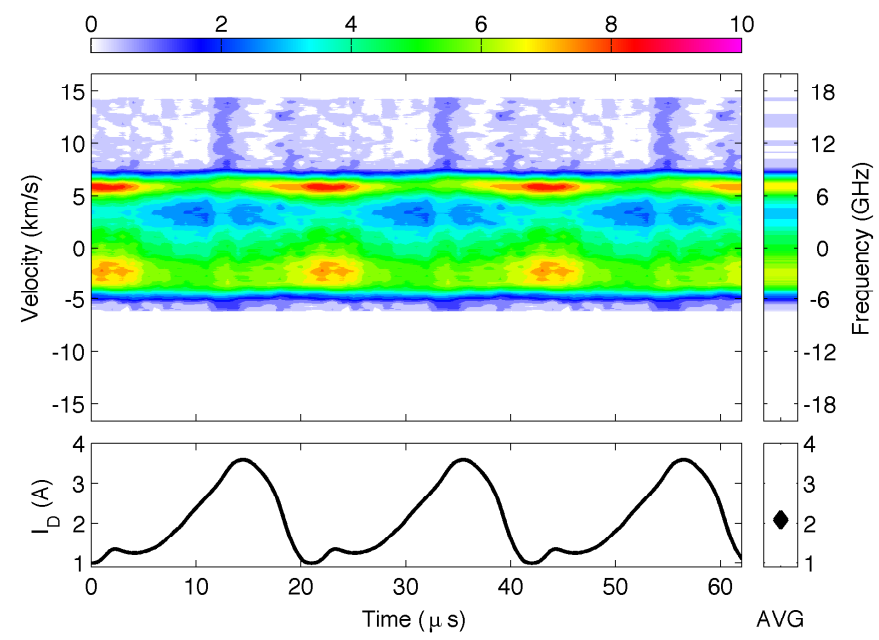

(a) $(x, z)=(0,74)$

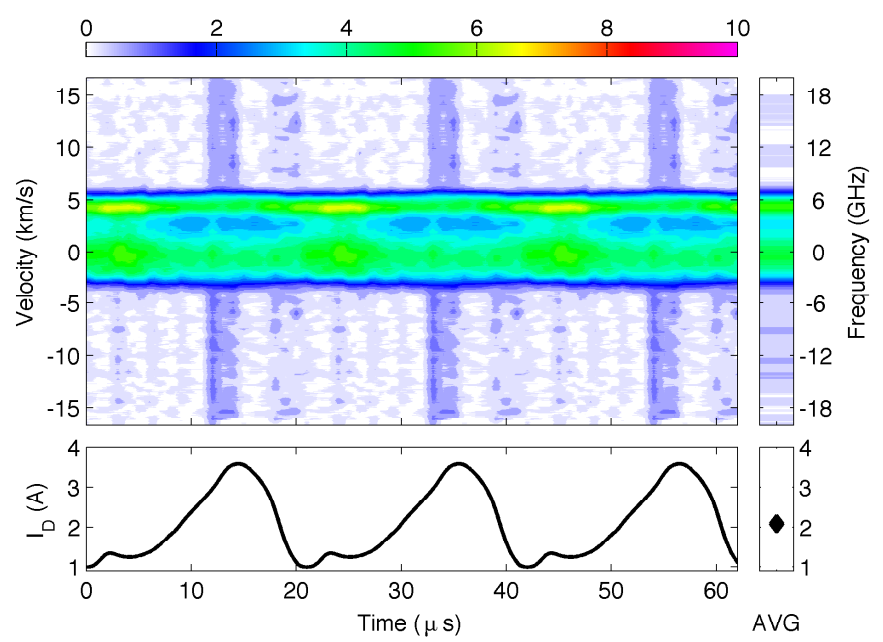

(b) $(x, z)=(0,100)$

FIG. 9: Contour plots of the time-resolved LIF lineshapes, displaying the true signal intensity (V), at two points along the main axis in the far-field plume of the BHT-600. The IVDFs are fairly constant in time. The data is repeated over three discharge current cycles (bottom panel) for visual effect. The corresponding time-averaged values appear in the boxes at right.

terval, studies of the dynamics within the channel show that the acceleration zone is furthest downstream and the electric field structure is widest. ${ }^{15}$ While it is unclear which side of the channel corresponds to each axial LIF peak, this effect is observed in all the time-resolved axial traces along the line $x=0$. The shift back from right to left side dominant occurs a few microseconds earlier when moving closer to the thruster.

Now examining the traces obtained closest to the thruster (Fig. 8b), one observes further multiple populations in the axial ion velocity distributions. The point at $z=2 \mathrm{~mm}$ is very close to the ceramic nose cone (see Fig. 1) and the significant number of ions traveling in the negative direction is likely due to the sheath that has formed on this surface. (Alternately, if any exciting laser light is specularly reflected, this would reverse the Doppler shift interaction with ions and produce a mirror image of the IVDF in negative velocity space. However, the dull ceramic should diffusely scatter the majority of the incoming beam.) $z=10 \mathrm{~mm}$ seems to be a sort of transition region, where well-formed IVDF peaks emanate both downstream and upstream from this location. A less well-defined axial population appears to follow the main one downstream from this point, with an average velocity about $5 \mathrm{~km} / \mathrm{s}$ slower.

More information about these ions may be gleaned from the time-resolved traces displayed in Fig. 11. For better visualization of the multiple populations, these contour plots show LIF scans normalized by the integrated intensity of each trace, with each panel normalized by the overall maximum value. The data is repeated over three discharge current cycles (bottom box in each panel) to evoke the periodic nature of the oscillation. Note that in contrast to Fig. 9, a high signal level now represents a narrow IVDF, not peak LIF intensity, although the peak intensity does also modulate at these locations as seen in the third column of Fig. 7.

Panels (b), (d), (e), and (f) of Fig. 9 illustrate the time-resolved axial ion dynamics underlying the timeintegrated traces taken along the main axis through the central jet from $z=10 \mathrm{~mm}$ to $z=25 \mathrm{~mm}$ (Fig. $8 \mathrm{~b}$ ). Most striking is that both ion populations show strong modulations in velocity over the breathing mode cycle, and that they progress opposite in phase to one another (behavior seen previously in a lower power Z-70 thruster $\left.{ }^{21}\right)$. As evidenced from the spatial maps of Figs. $6 \mathrm{c}$ and 7 (first column), these modulations progress at a different phase relative to the discharge current than those of the channel and near field. As described earlier, this is most likely due to the finite transit time of ions between their origin in the channel and the measurement locations. This provides compelling evidence for both residual ionization of neutrals in the central jet, and a moving potential front in this center region of the thruster linked to the breathing mode.

As seen in Fig. 11e, the low velocity population originates in the transition region previously identified near $z=10 \mathrm{~mm}$. Here, the dominant population appears to be the residual ions (some of which have obtained a negative velocity). Since ions are born with approximately zero velocity, these would be more likely affected by the local potential of the sheath on the thruster surface, turning upstream. Relatively fewer accelerated ions reach this point, which would imply a large divergence angle from the channel $\left(\sim 70^{\circ}\right)$. The time-averaged traces upstream of $z=10 \mathrm{~mm}$ are then likely comprised of these residual ions. Downstream of $z=10 \mathrm{~mm}$ the residual ions continue to accelerate, and after traveling $15 \mathrm{~mm}$ 

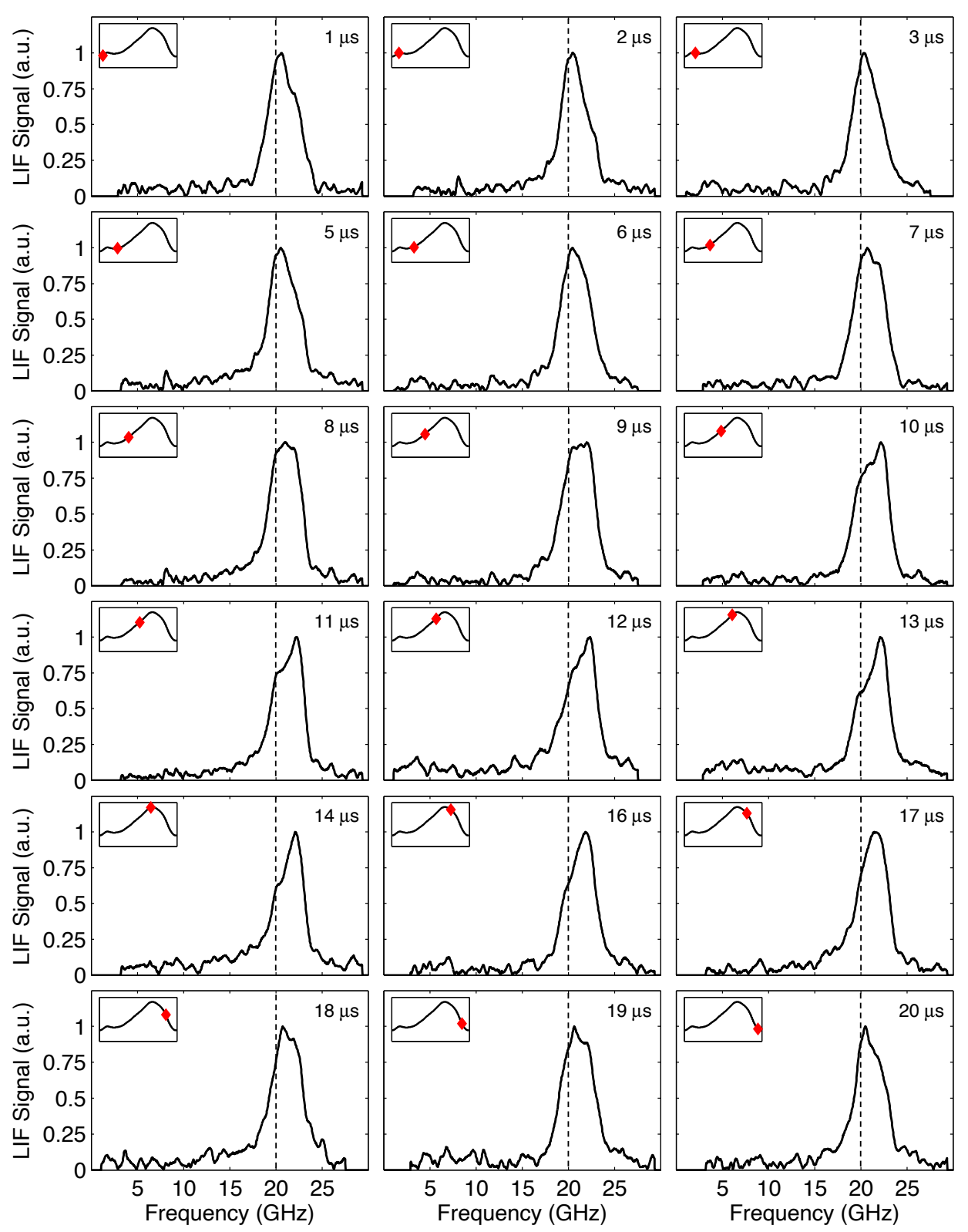

FIG. 10: Collection of individual time-resolved axial LIF traces at $(x, z)=(0,74)$, showing axial asymmetry along the central jet. Each trace is normalized to the peak value. The variation of the peak intensity can be found in the middle panel of Fig. 5c. The boxes at the upper left of each panel show the instantaneous value of the discharge current at each time frame. The vertical dotted line at $20 \mathrm{GHz}$ is provided to guide the eye.

downstream, the low velocity population shows strong modulations between 4 and $12 \mathrm{~km} / \mathrm{s}$. Here, the IVDF of the low velocity population is narrower than might be expected from the broad features seen in the timeintegrated traces, demonstrating how a strongly oscillating IVDF can act to broaden a time-averaged measurement (behavior also seen previously in the Z-70 thruster). Off-center at $x=-4 \mathrm{~mm}$, the traces become somewhat noisier but the same patterns persist.

The similarities between the low axial velocity population dynamics seen here and those from the Z- $70^{21}$ suggest comparable breathing mode physics may be behind the observed behavior (with a delay of about $6-8 \mathrm{~s}$ between modulations occurring at the channel and within the central jet due to the ion transit time). When the accelerating potential is farthest upstream in the channel, the main beam of accelerated ions has attained a faster velocity by the exit plane. Residual ions born in the plume at this time see only a small tail of the potential drop, and thus attain their slowest velocities during this interval. This is reflected in the LIF data of Fig. 11, where the dominant population reaches peak velocity (and LIF intensity) during the ramp up in discharge current and the residual ion population is near 


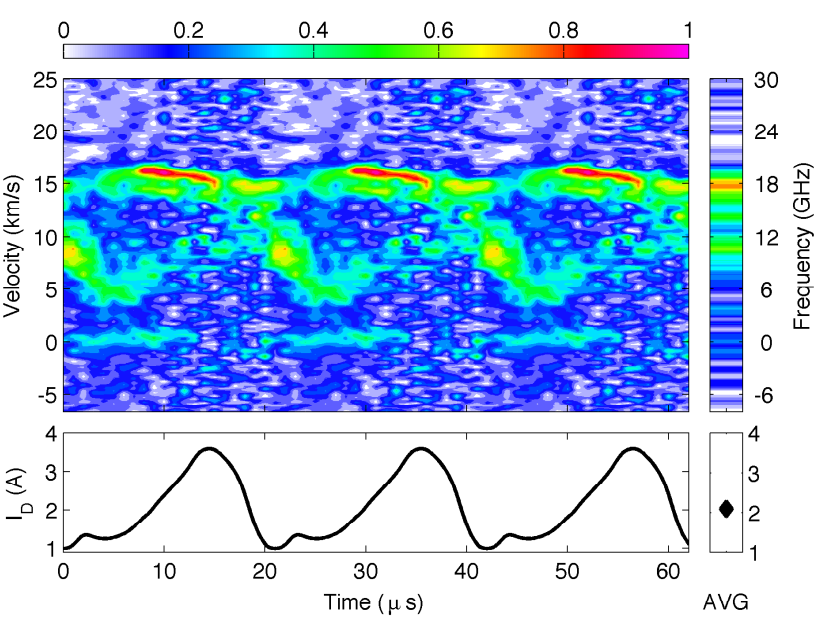

(a) $(x, z)=(-4,25)$

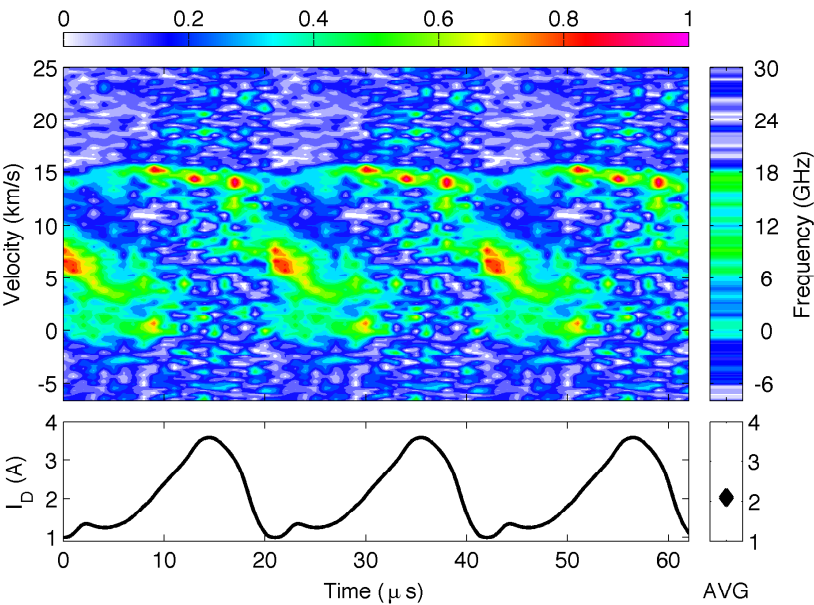

(c) $(x, z)=(-4,20)$

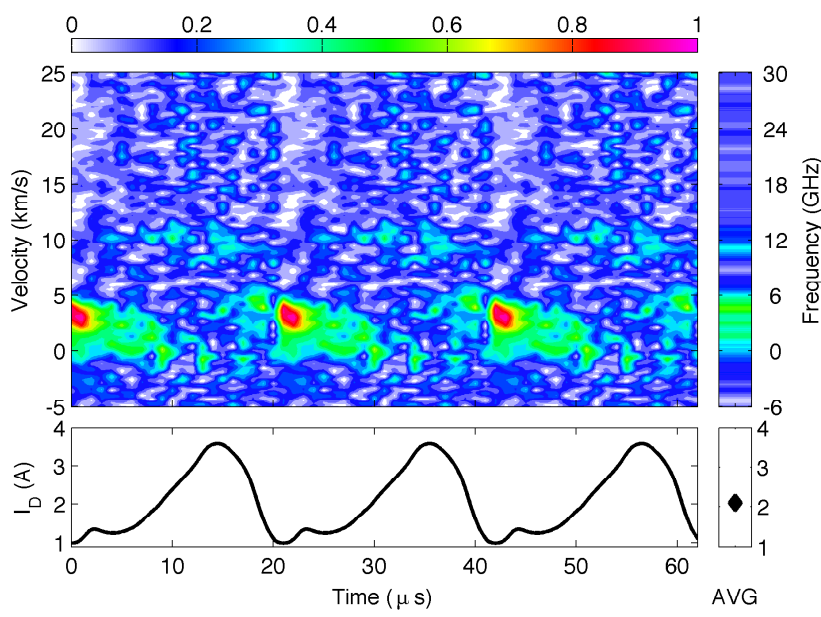

(e) $(x, z)=(0,10)$

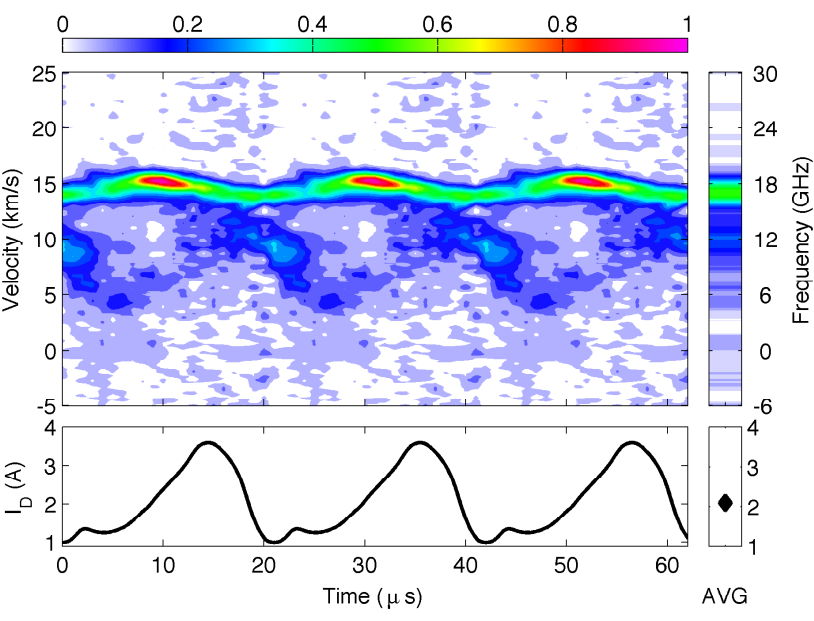

(b) $(x, z)=(0,25)$

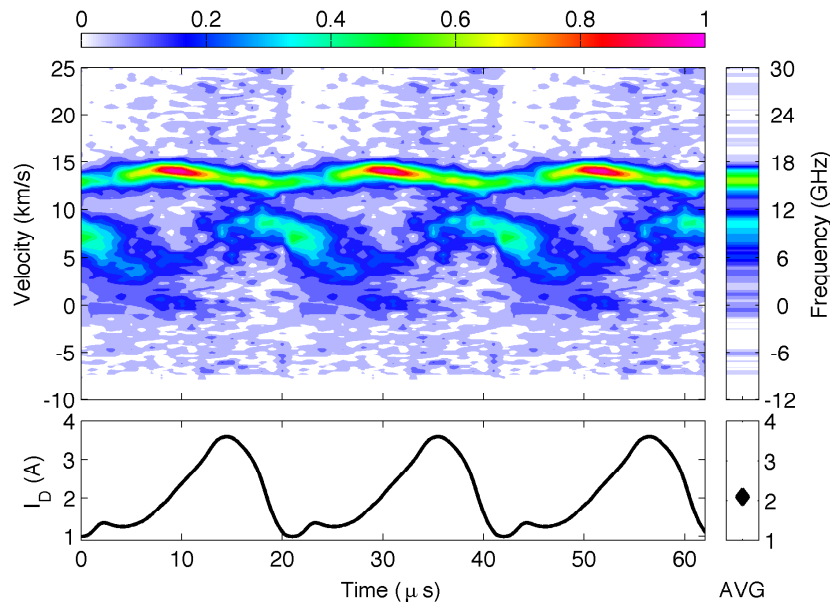

(d) $(x, z)=(0,20)$

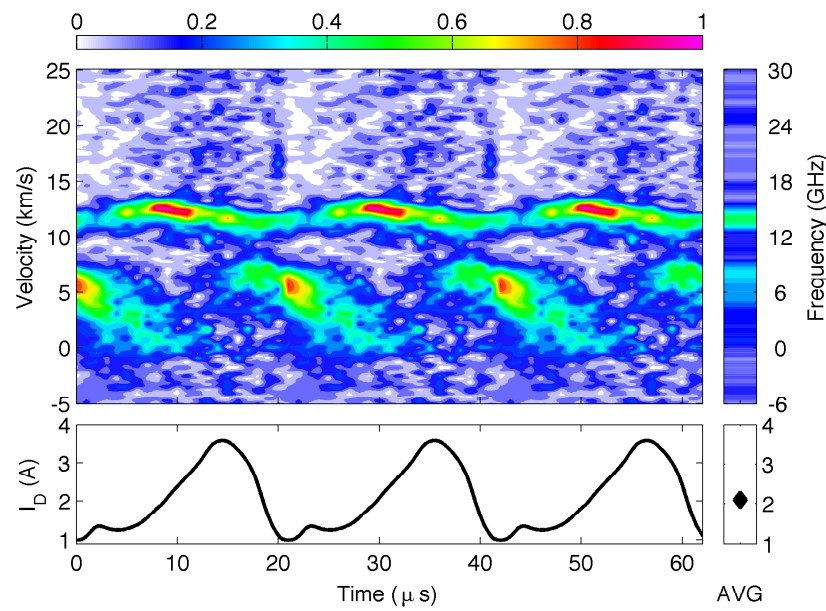

(f) $(x, z)=(0,15)$

FIG. 11: Time-resolved axial LIF contour plots showing multiple ion populations throughout the BHT-600 central jet. Each scan (vertical strip) is normalized by the integrated signal, with each panel normalized by the overall maximum. The corresponding time-averaged LIF and discharge current traces are reproduced to the right and below each panel, respectively. The data is repeated over three cycles for visual effect. Data is obtained from (a) $(x, z)=(-4,25),(\mathrm{b})(x, z)=(0,25),(\mathrm{c})(x, z)=(-4,20),(\mathrm{d})(x, z)=(0,20),(\mathrm{e})(x, z)=(0,10),(\mathrm{f})(x, z)=(0,15)$. 
its point of lowest velocity and LIF intensity. In contrast, when the ionization zone is furthest downstream, primary ions have only been partly accelerated by the time they reach the exit plane. Now, residual ions born in the plume see a much larger portion of the potential drop tail, experiencing a boost in their velocities during this time. This occurs in Fig. 11 just after the discharge current begins to fall from its peak, when the residual population is at maximum velocity and the dominant population reaches its minimum. Since ions originating in the channel experience the majority of the potential drop no matter when they are born, the relative strength of the velocity modulations are weaker than those of the low velocity ions created externally to the thruster. From $\mathrm{z}=15 \mathrm{~mm}$ to $\mathrm{z}=25 \mathrm{~mm}$, both populations maintain a consistent offset of $\sim 5 \mathrm{~km} / \mathrm{s}$ as they are accelerated, suggesting the presence of a local electric field.

\section{Other Low Velocity Axial Populations}

Other secondary axial populations with low velocity are observed in the inner plume area between the exit plane and the central jet, and immediately beyond the exit plane. No significant low velocity populations are found in the outer plume area $|x, y|>28 \mathrm{~mm}$ past about $6 \mathrm{~mm}$ downstream of the exit plane. Some representative time-resolved LIF contours appear in Fig. 12, in both the $X Z$ and $Y Z$ planes. Each scan is normalized by the integrated signal intensity as in Fig. 11; note that in many of these positions overall signal strength (and thus metastable ion density) is low.

Many measurement locations show the same patterns observed in the central jet: the emergence of a low velocity population during the discharge current trough with temporal modulations progressing opposite that of the main population. Just beyond the channel exit at $(y, z)=(-36,2) \mathrm{mm}$ and $(x, z)=(-20,2) \mathrm{mm}$, some negative ion velocities are observed in the low velocity populations. Again, this is attributed to the sheath forming on thruster surfaces. At the inner edge of the channel, Fig. 12d, there are essentially no temporal modulations in either ion population. In contrast, at the outer edge of the channel (Fig. 12c) the counter-streaming ion population shows a clear oscillation at the breathing mode frequency between around $0 \mathrm{~km} / \mathrm{s}$ during the discharge current trough and $-5 \mathrm{~km} / \mathrm{s}$ at the current peak. Previous work ${ }^{15}$ indicates that the discharge current increases as the ionization front moves upstream in the channel, consuming neutral propellant and generating ions. Thus, the negatively directed low velocity ions are most accelerated by the sheath potential when the main ionization front moves upstream, away from this region. The effect is reduced, then neutralized completely, as the breathing mode potential front returns downstream before the start of the next ionization cycle. These time-resolved LIF measurements reveal new interesting features in the ion dynamics throughout the thruster domain.

\section{CONCLUSION}

Time-resolved continuous wave LIF measurements of metastable ion velocities in the near-field of a BHT-600 Hall thruster have revealed details about the dynamical behavior and structure. Data is acquired using a parallelized sample-hold scheme with multiple data acquisition channels enabling a collection of a large dataset of 2-D, time-resolved information throughout the plume at a single operating condition. Measurements are synchronized with the $44-49 \mathrm{kHz}$ breathing mode oscillation characteristic of these thrusters.

Building on the detailed prior survey ${ }^{15}$ of ion velocity distributions throughout the discharge channel which revealed IVDFs with a very strong intensity and velocity modulation in the zone of primary ion acceleration, these near-field studies indicate that the majority of ion acceleration is complete by the first centimeter into the plume, and the magnitude of velocity modulations on top of the average value lessens moving downstream. Clear modulation cycles are still apparent, however, enabling the velocity and intensity modulations at many points throughout the plume to be compared with the discharge current cycle via a cross-correlation. In general, the lag time between the intensity/velocity modulations and the current trace increases with distance away from the thruster. The increases in lag time is shown to roughly track the ion transit time between the thruster and measurement point. Plotting spatial maps of the normalized oscillating quantities throughout the plume reveals wave-like structures that again propagate approximately at the rate of ion motion throughout the plume.

An examination of the central jet reveals asymmetry in both the radial and axial velocity distribution functions, with multiple ion populations observed throughout. The presence of low velocity axial populations lends support to the theory that the central jet is an avenue for residual ionization of neutrals traversing the near-field plume. These could contribute to the significant LIF signal near zero radial velocity, in addition to the two main beams crossing from opposite sides of the channel. The opposite velocity modulations of the low and high axial populations, as seen in a lower power (Z-70) thruster, ${ }^{21}$ is reminiscent of an axially oscillating potential hill that strongly accelerates residual ions in the plume during part of the discharge cycle. Other slow velocity populations are observed near the channel exit plane, and in the intervening space between the channel and main thruster axis, many with the same characteristic opposite modulation trend. This provides compelling evidence that residual ionization of neutral atoms is responsible for the low velocity ion populations observed in both this BHT-600 as well as the other systems. Further study is required to confirm this theory and study the effects of chamber background pressure and operating condition on the low velocity populations. The extent to which these populations and their dynamics affect thruster operation and performance in space depends on the origin of the 


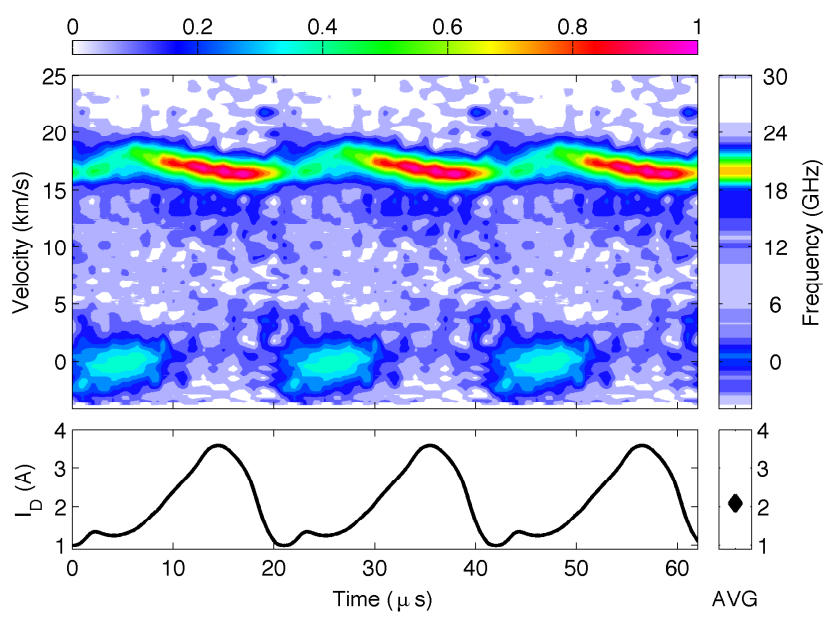

(a) $(x, z)=(-20,10)$

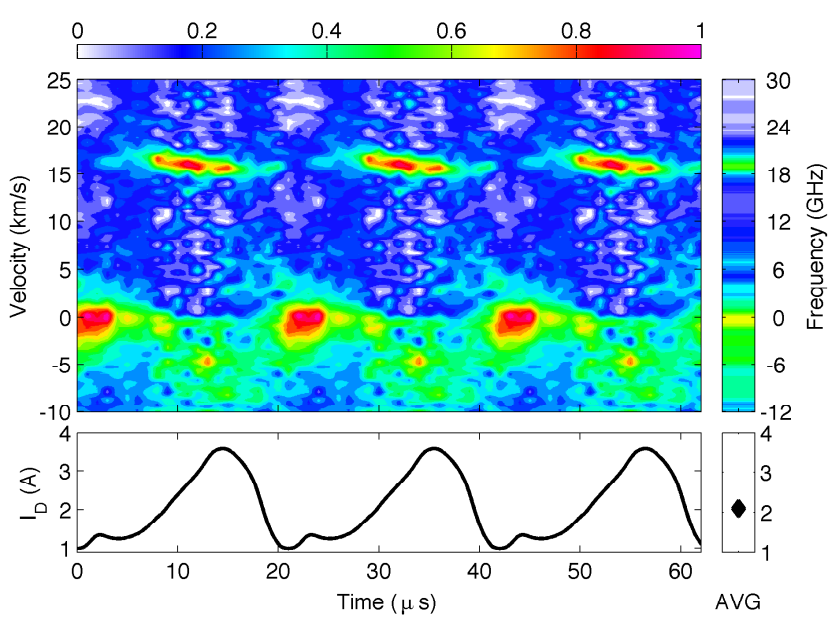

(c) $(y, z)=(-36,2)$

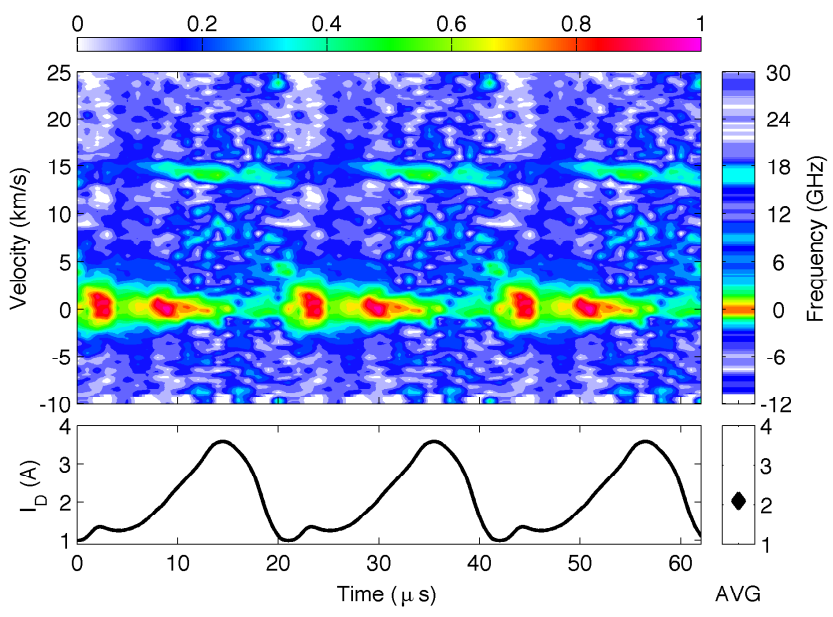

(b) $(x, z)=(-12,10)$

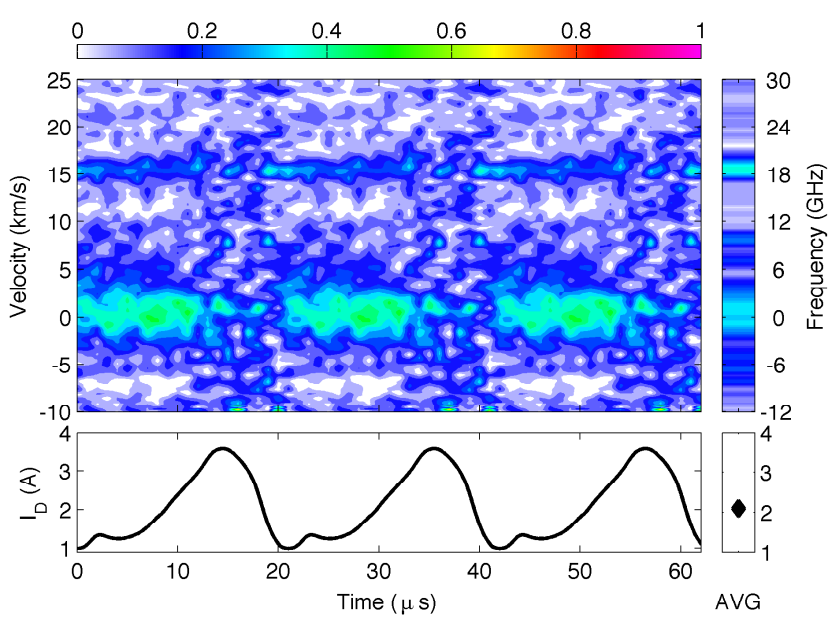

(d) $(y, z)=(-20,2)$

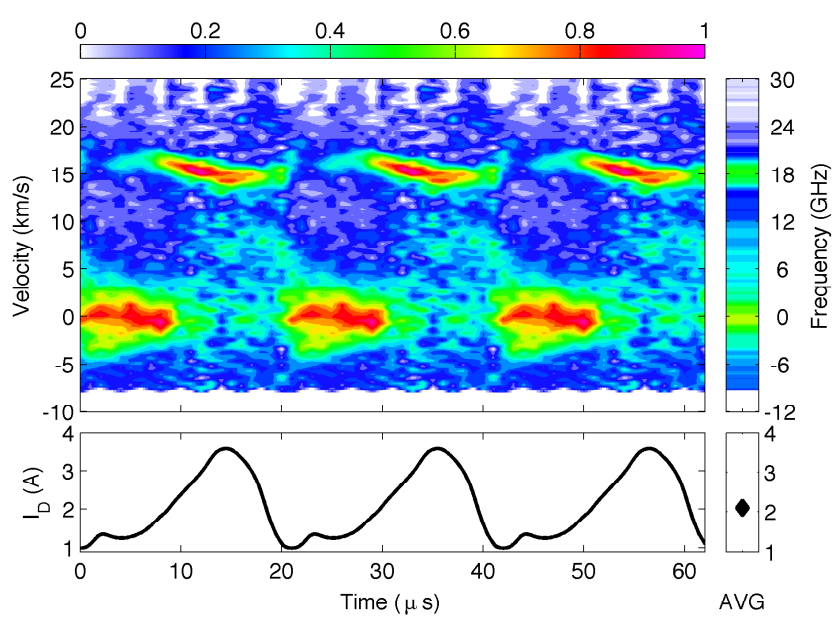

(e) $(x, z)=(-20,2)$

FIG. 12: Time-resolved axial LIF contours showing multiple ion velocity populations throughout the near-field plume. The normalization is the same as in Fig. 11. The data is obtained from: (a) $(x, z)=(-12,10)$, (b) $(x, z)=(-12,10),(\mathrm{c})(y, z)=(-36,2),(\mathrm{d})(y, z)=(-20,2),(\mathrm{e})(x, z)=(-20,2)$. 
residual neutrals (unionized propellant vs. background pressure from an imperfect ground test chamber) and is worth further examination for space qualified systems.

\section{ACKNOWLEDGEMENTS}

This work is sponsored by the U.S. Air Force Office of Scientific Research with Dr. M. Birkan as program manager. C.Y. acknowledges support from the DOE NNSA Stewardship Science Graduate Fellowship under contract DE-NA0002135 and the Stanford Graduate Fellowship.

\section{DATA AVAILABILITY STATEMENT}

The datasets analyzed for this study can be made available upon request.

\section{REFERENCES}

${ }^{1}$ M. A. Cappelli, "The Hall effect and rocket flight," Physics Today 62, 76 (2009).

${ }^{2} \mathrm{~V}$. Kim, "Main physical features and processes determining the performance of stationary plasma thrusters," Journal of Propulsion and Power 14, 736-743 (1998).

${ }^{3}$ J.-P. Boeuf and L. Garrigues, "Low frequency oscillations in a stationary plasma thruster," Journal of Applied Physics 84, 3541 (1998).

${ }^{4} \mathrm{~S}$. Barral and E. Ahedo, "Low-frequency model of breathing oscillations in Hall discharges," Physical Review E 79, 046401 (2009).

${ }^{5}$ J. M. Fife, M. Martinez-Sanchez, and J. Szabo, "A numerical study of low-frequency discharge oscillations in Hall thrusters," in Proceedings of the 33rd AIAA/ASME/SAE/ASEE Joint Propulsion Conference \& Exhibit (Seattle, WA, 1997).

${ }^{6} \mathrm{~S}$. Barral and Z. Peradzynski, "A new breath for the breathing mode," in Proceedings of the 31st International Electric Propulsion Conference, IEPC-2009-070 (Ann Arbor, MI, 2009).

${ }^{7}$ K. Dannenmayer, P. Kudrna, M. Tichỳ, and S. Mazouffre, "Time-resolved measurement of plasma parameters in the farfield plume of a low-power hall effect thruster," Plasma Sources Science and Technology 21, 055020 (2012).

${ }^{8}$ K. Dannenmayer, S. Mazouffre, P. Kudrna, and M. Tichỳ, "The time-varying electron energy distribution function in the plume of a hall thruster," Plasma Sources Science and Technology 23, 065001 (2014).

${ }^{9}$ R. B. Lobbia, A time-resolved investigation of the Hall thruster breathing mode, Ph.D. thesis, University of Michigan (2010).

${ }^{10}$ M. Touzeau, M. Prioul, S. Roche, N. Gascon, C. Pérot, F. Darnon, S. Béchu, C. Philippe-Kadlec, L. Magne, P. Lasgorceix, et al., "Plasma diagnostic systems for Hall-effect plasma thrusters," Plasma Physics and Controlled Fusion 42, B323 (2000).

${ }^{11}$ A. Bouchoule, C. Philippe-Kadlec, M. Prioul, F. Darnon, M. Lyszyk, L. Magne, D. Pagnon, S. Roche, M. Touzeau, S. Béchu, et al., "Transient phenomena in closed electron drift plasma thrusters: Insights obtained in a French cooperative program," Plasma Sources Science and Technology 10, 364 (2001).

${ }^{12}$ M. R. Nakles, M. R. Holmes, and W. A. Hargus Jr., "An investigation into the spectral imaging of Hall thruster plumes," in Proceedings of the 34 th International Electric Propulsion Conference, IEPC-2015-416 (Hyogo-Kobe, Japan, 2015).

${ }^{13}$ D. Liu, R. E. Huffman, R. D. Branam, and W. A. Hargus, Jr., "Ultrahigh-speed imaging of Hall-thruster discharge oscillations with krypton propellant," IEEE Transactions on Plasma Science 39, 2926-2927 (2011)

${ }^{14}$ V. Vial, S. Mazouffre, M. Prioul, D. Pagnon, and A. Bouchoule, "Ccd images of hall effect thruster plume dynamics after ultrafast current ignition," IEEE Transactions on Plasma Science 33, 524525 (2005).

${ }^{15}$ C. Young, A. Lucca Fabris, N. MacDonald-Tenenbaum, W. Hargus, and M. Cappelli, "Time-resolved laser-induced fluorescence diagnostics for electric propulsion and their application to breathing mode dynamics," Plasma Sources Science and Technology 27, 094004 (2018).

${ }^{16}$ C. V. Young, Dynamics of Plasma Discharges Used for Space Propulsion, Ph.D. thesis, Stanford University (2016).

${ }^{17}$ S. Mazouffre, L. Grimaud, S. Tsikata, K. Matyash, and R. Schneider, "Rotating spoke instabilities in a wall-less hall thruster: Experiments," Plasma Sources Science and Technology 28, 054002 (2019).

${ }^{18}$ C. Durot, A. Gallimore, and T. Smith, "Validation and evaluation of a novel time-resolved laser-induced fluorescence technique," Review of Scientific Instruments 85, 013508 (2014).

${ }^{19}$ A. Diallo, S. Keller, Y. Shi, Y. Raitses, and S. Mazouffre, "Timeresolved ion velocity distribution in a cylindrical Hall thruster: Heterodyne-based experiment and modeling," Review of Scientific Instruments 86, 033506 (2015).

${ }^{20} \mathrm{~J}$. Vaudolon, L. Balika, and S. Mazouffre, "Photon counting technique applied to time-resolved laser-induced fluorescence measurements on a stabilized discharge," Review of Scientific Instruments 84, 073512 (2013)

${ }^{21}$ A. Lucca Fabris, C. V. Young, and M. A. Cappelli, "Timeresolved laser-induced fluorescence measurement of ion and neutral dynamics in a Hall thruster during ionization oscillations," Journal of Applied Physics 118, 233301 (2015).

${ }^{22}$ C. J. Durot, M. P. Georgin, and A. D. Gallimore, "Timeresolved laser-induced fluorescence measurements in the plume of a 6-kW Hall thruster," in Proceedings of the 34th International Electric Propulsion Conference, IEPC-2015-399 (HyogoKobe, Japan, 2015)

${ }^{23}$ V. Chaplin, R. Lobbia, A. Lopez Ortega, I. Mikellides, R. Hofer J. Polk, and A. Friss, "Time-resolved ion velocity measurements in a high-power hall thruster using laser-induced fluorescence with transfer function averaging," Applied Physics Letters 116, 234107 (2020)

${ }^{24}$ Busek Company Inc., BHT-600 Busek Hall effect thruster datasheet, [Online]. Available: http://www.busek.com/index_htm_files/70000701\%20BHT-600 $\% 20$ Data\%20Sheet\%20Rev-.pdf. [2021, May 26]. Natick, MA. (2016).

25 A. E. Gonzales, J. W. Koo, and W. A. Hargus, Jr., "Comparison of numerical and experimental time-resolved near-field Hal thruster plasma properties," IEEE Transactions on Plasma Science 42, 806-812 (2014).

${ }^{26}$ N. A. MacDonald, M. A. Cappelli, and W. A. Hargus Jr., "Timesynchronized continuous wave laser-induced fluorescence axial velocity measurements in a diverging cusped field thruster," Journal of Physics D: Applied Physics 47, 115204 (2014).

${ }^{27}$ I. Romadanov, Y. Raitses, A. Diallo, K. Hara, I. Kaganovich and A. Smolyakov, "On limitations of laser-induced fluorescence diagnostics for xenon ion velocity distribution function measurements in hall thrusters," Physics of Plasmas 25, 033501 (2018).

${ }^{28}$ A. Lucca Fabris, C. V. Young, and M. A. Cappelli, "Excited state population dynamics of a xenon ac discharge," Plasma Sources Science and Technology 24, 055013 (2015).

${ }^{29}$ W. A. Hargus Jr. and C. S. Charles, "Near-plume laser-induced fluorescence velocity measurements of a medium power Hall thruster," Journal of Propulsion and Power 26, 135-141 (2010).

${ }^{30}$ P. H. Eilers, "A perfect smoother," Analytical Chemistry 75, 3631-3636 (2003)

${ }^{31}$ A. W. Smith and M. A. Cappelli, "Time and space-correlated plasma potential measurements in the near field of a coaxial hall plasma discharge," Physics of Plasmas 16, 073504 (2009). 
${ }^{32}$ V. Hruby, J. Monheiser, B. Pole, P. Rostler, and J. Kolencik, "Development of low power Hall thrusters," in 30th Plasmadynamic and Lasers Conference, Fluid Dynamics and Co-located Conferences, AIAA 99-3534 (Norfolk, VA, 1999).

${ }^{33} \mathrm{~A}$. Smith and M. Cappelli, "On the role of fluctuations, cathode placement, and collisions on the transport of electrons in the near-field of hall thrusters," Physics of plasmas 17, 093501 (2010).
${ }^{34}$ A. W. Smith and M. A. Cappelli, "Single particle simulations of electron transport in the near-field of hall thrusters," Journal of Physics D: Applied Physics 43, 045203 (2010).

${ }^{35}$ W. A. Hargus, Jr., "Laser-induced-fluorescence-derived Hall effect thruster ion velocity distribution visualization," IEEE Transactions on Plasma Science 39, 2918-2919 (2011). 
(a)
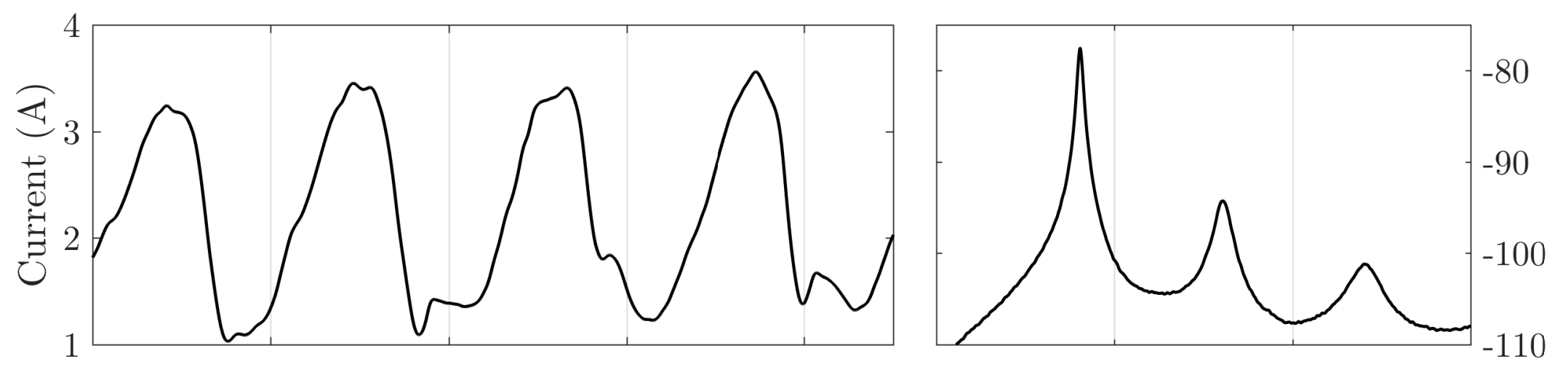

(b)
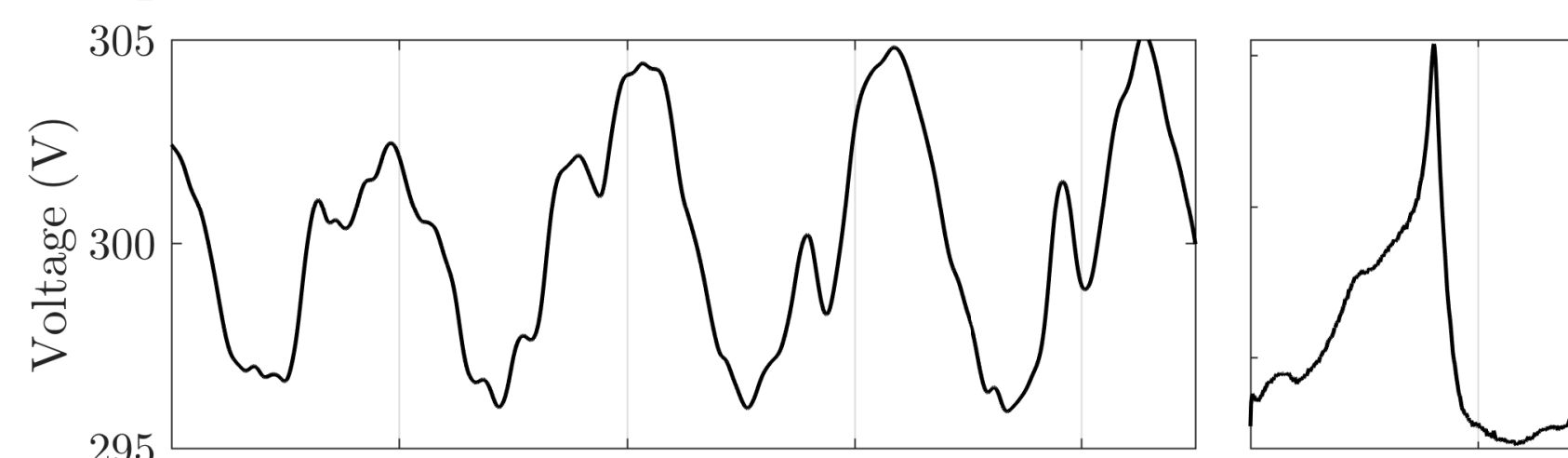

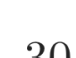

졸
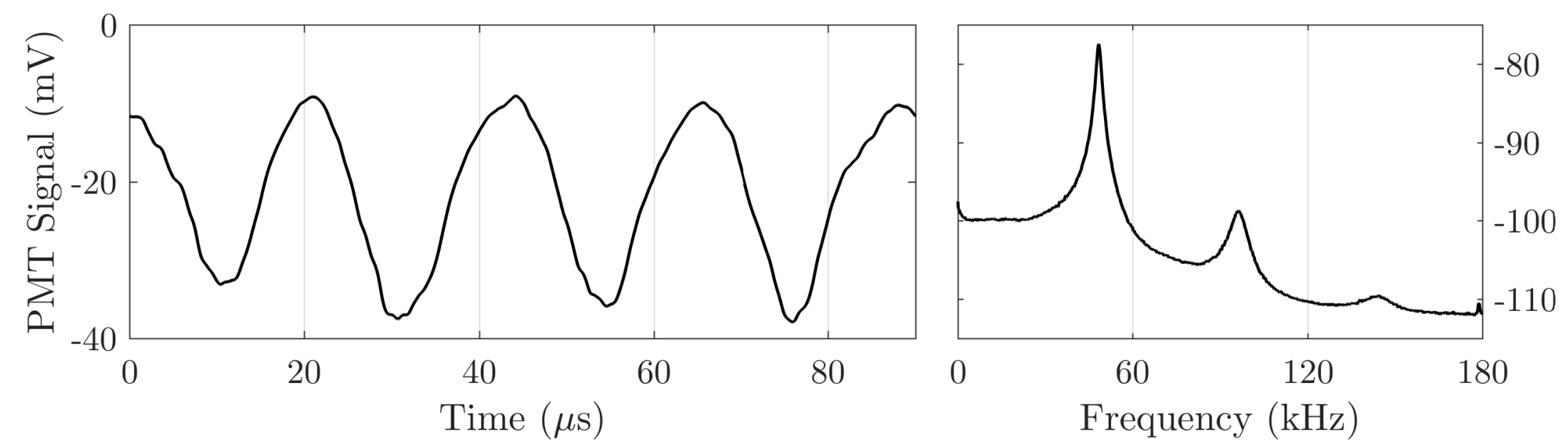


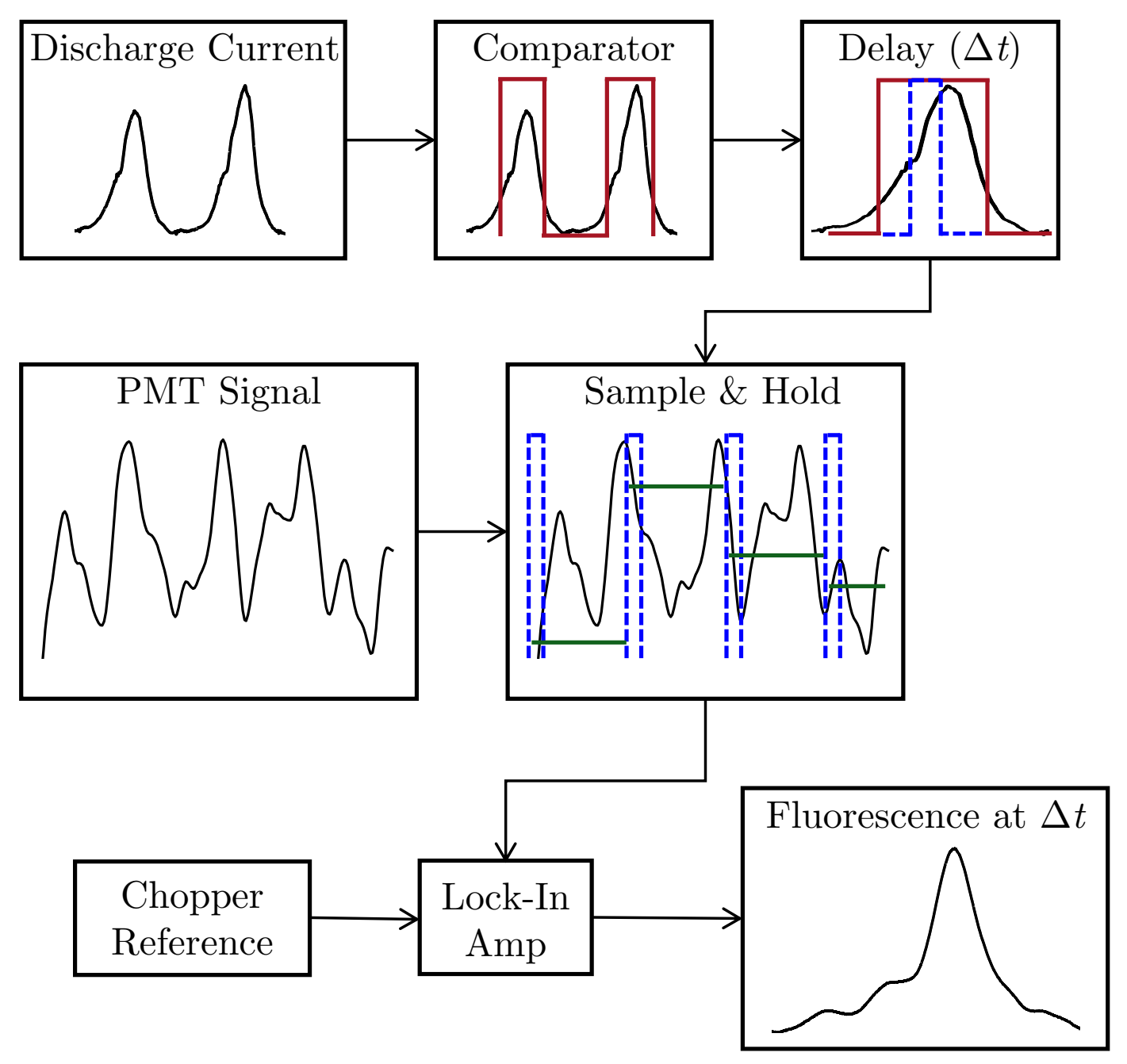


$\longrightarrow \mathrm{z}=6 \mathrm{~mm} \longrightarrow \mathrm{z}=54 \mathrm{~mm} \longrightarrow \mathrm{z}=100 \mathrm{~mm}$
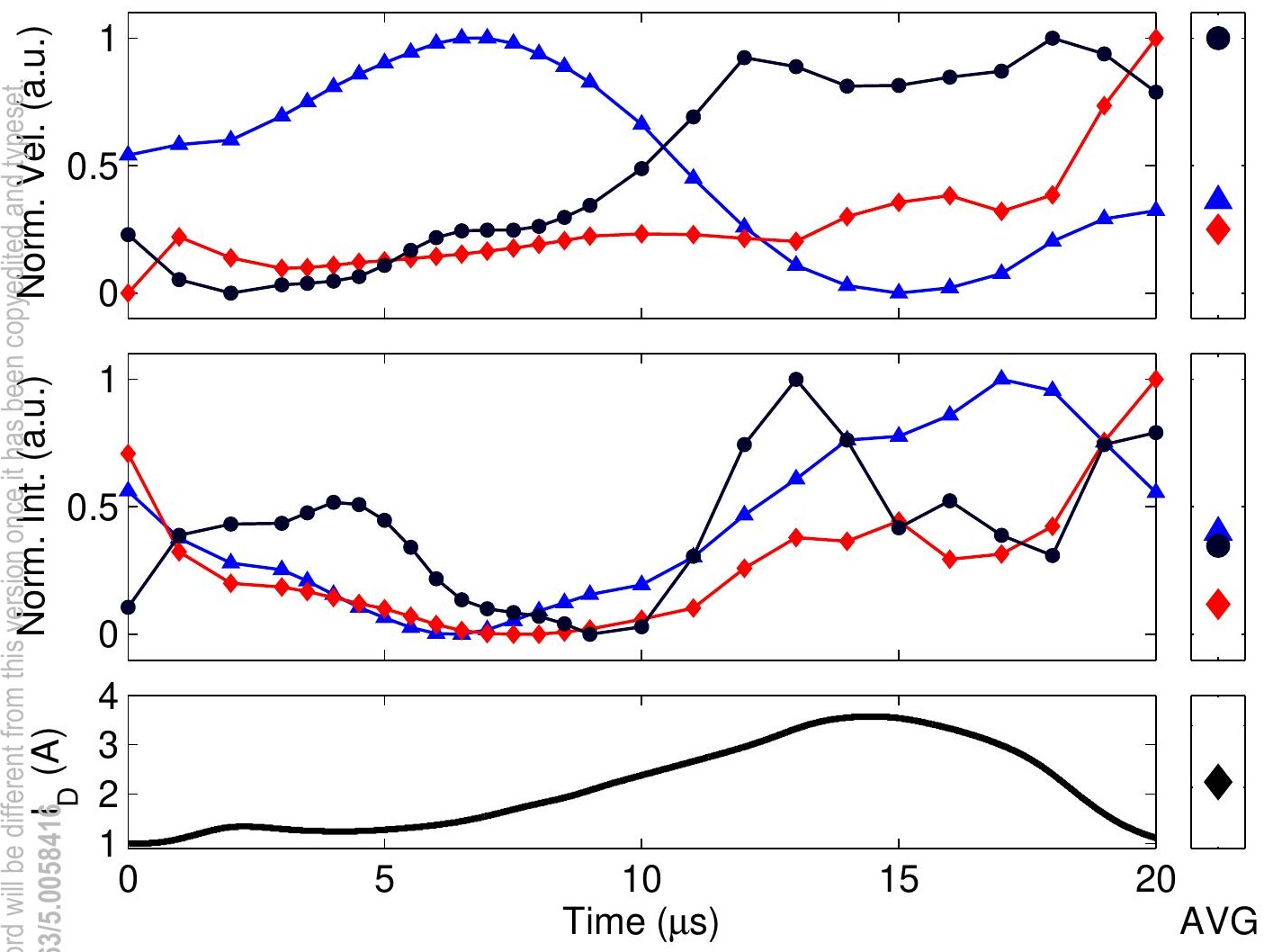
$\longrightarrow \mathrm{z}=6 \mathrm{~mm} \longrightarrow \mathrm{z}=54 \mathrm{~mm} \longrightarrow \mathrm{z}=100 \mathrm{~mm}$
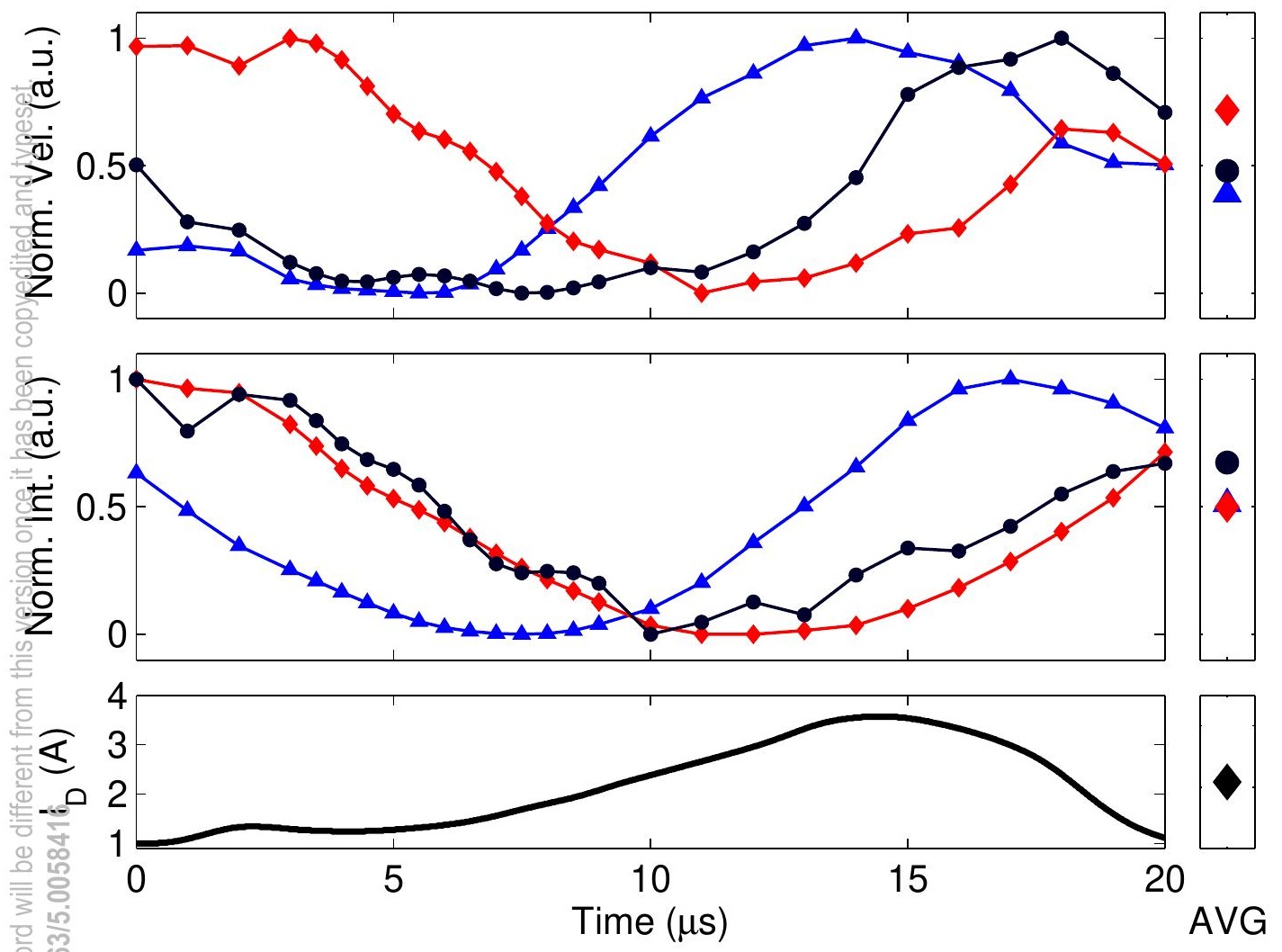
$\longrightarrow(\mathrm{x}, \mathrm{z})=(-28,6) \longrightarrow(\mathrm{x}, \mathrm{z})=(-16,34) \longrightarrow(\mathrm{x}, \mathrm{z})=(0,74)$
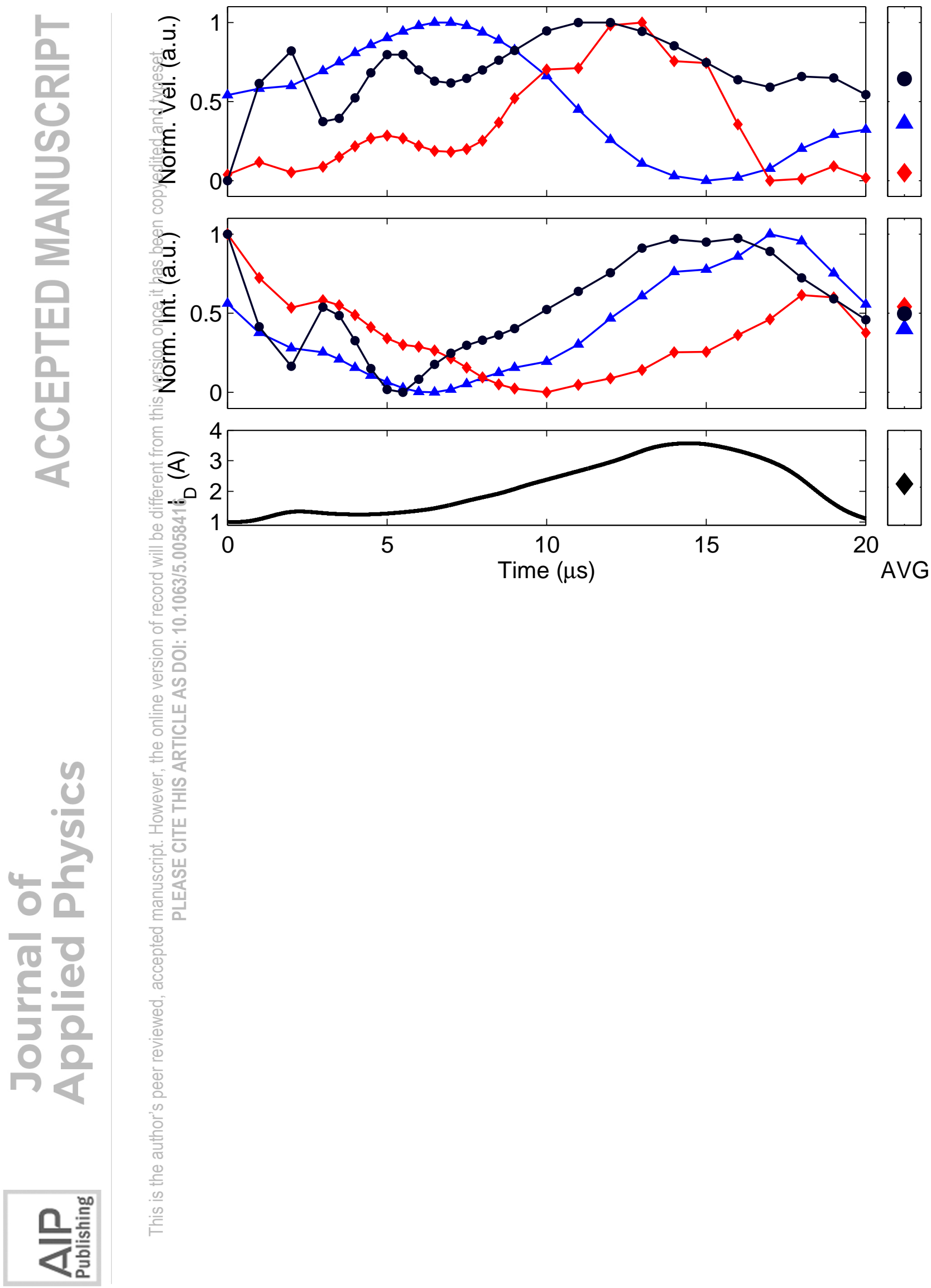
$\longrightarrow(x, z)=(-28,6) \longrightarrow(x, z)=(-16,34) \longrightarrow(x, z)=(0,74)$
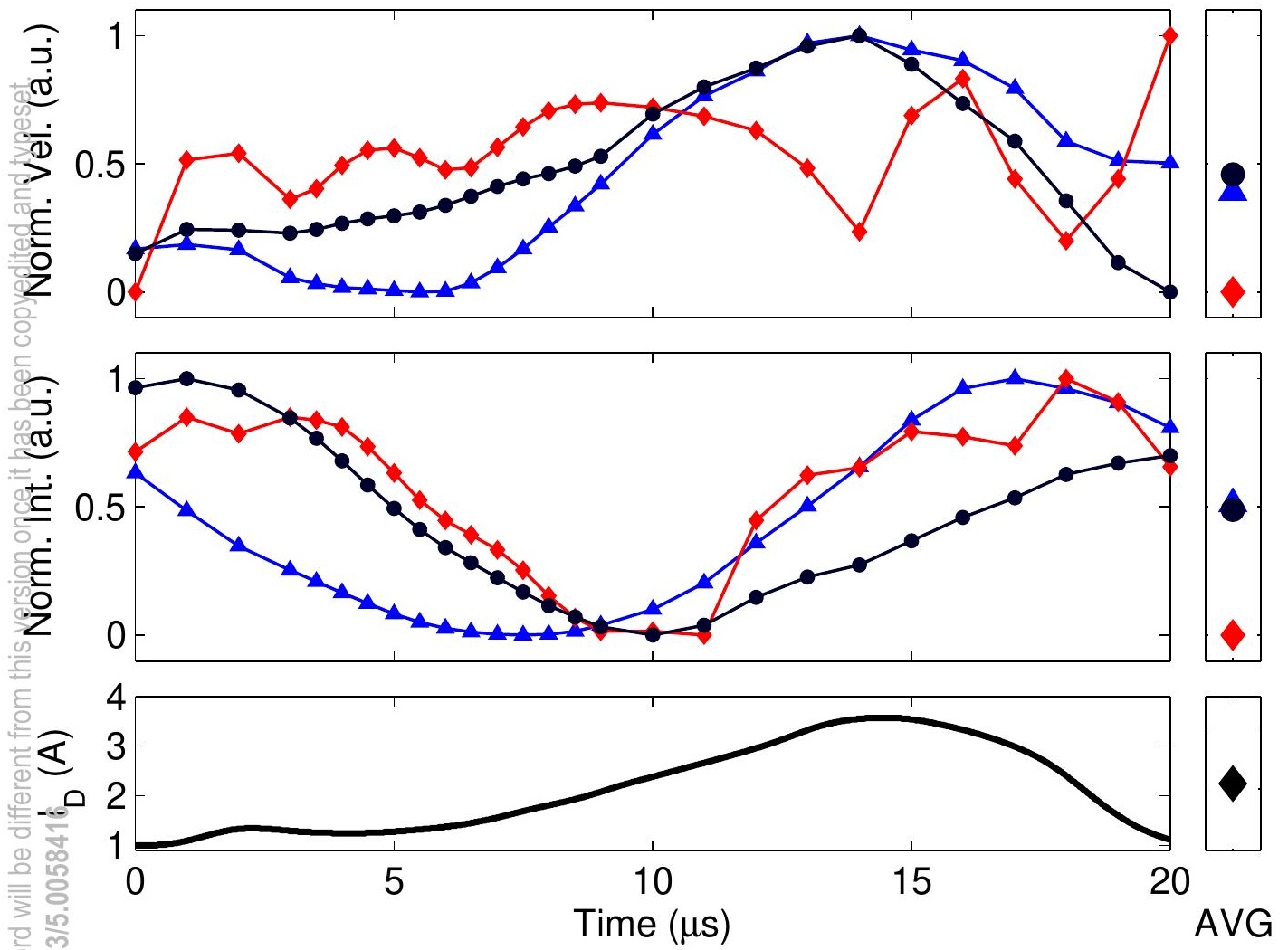


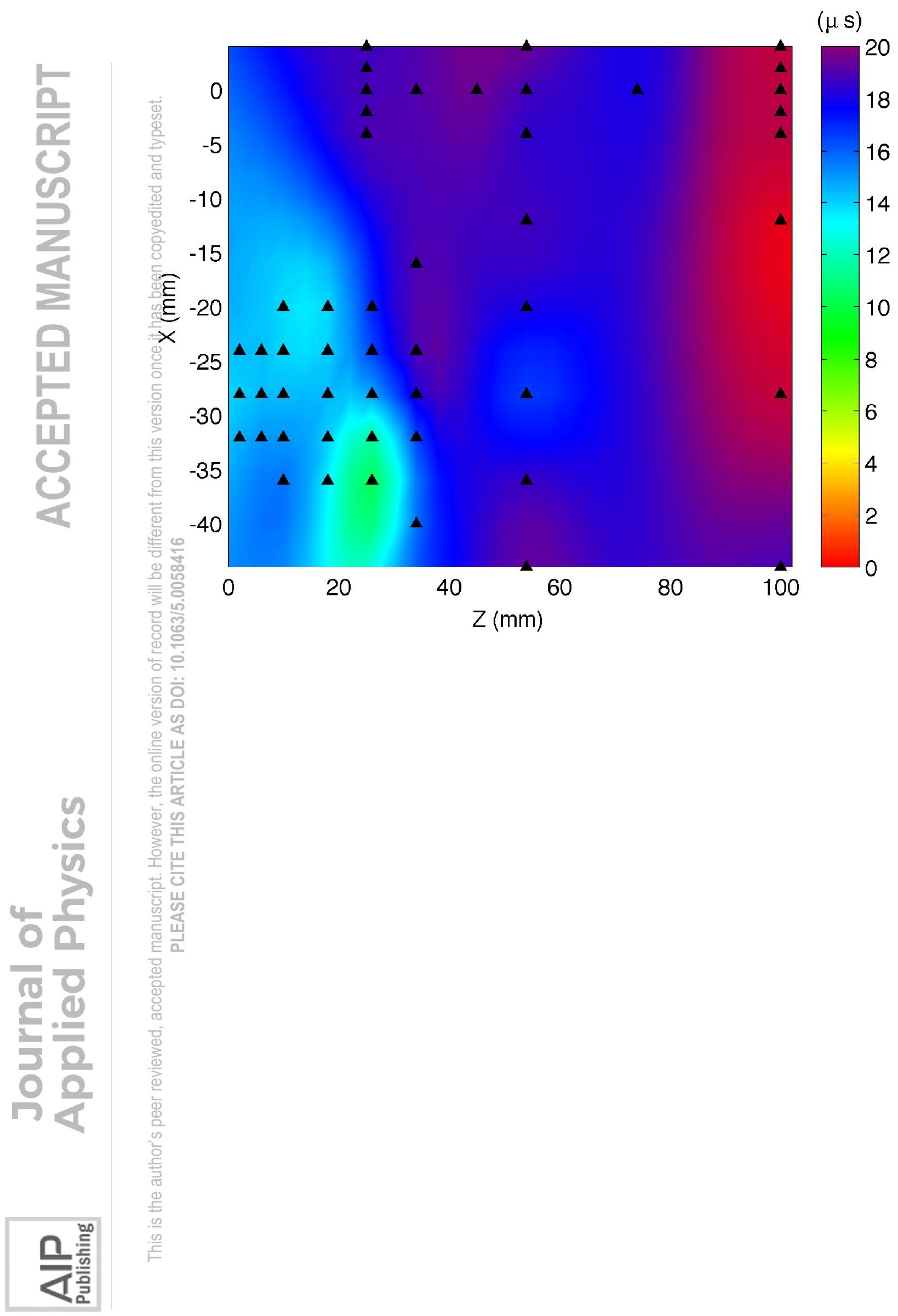


$(\mu s)$

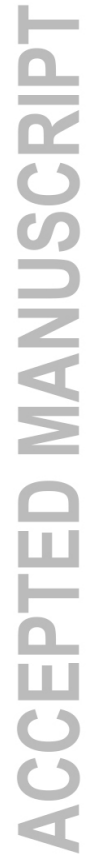

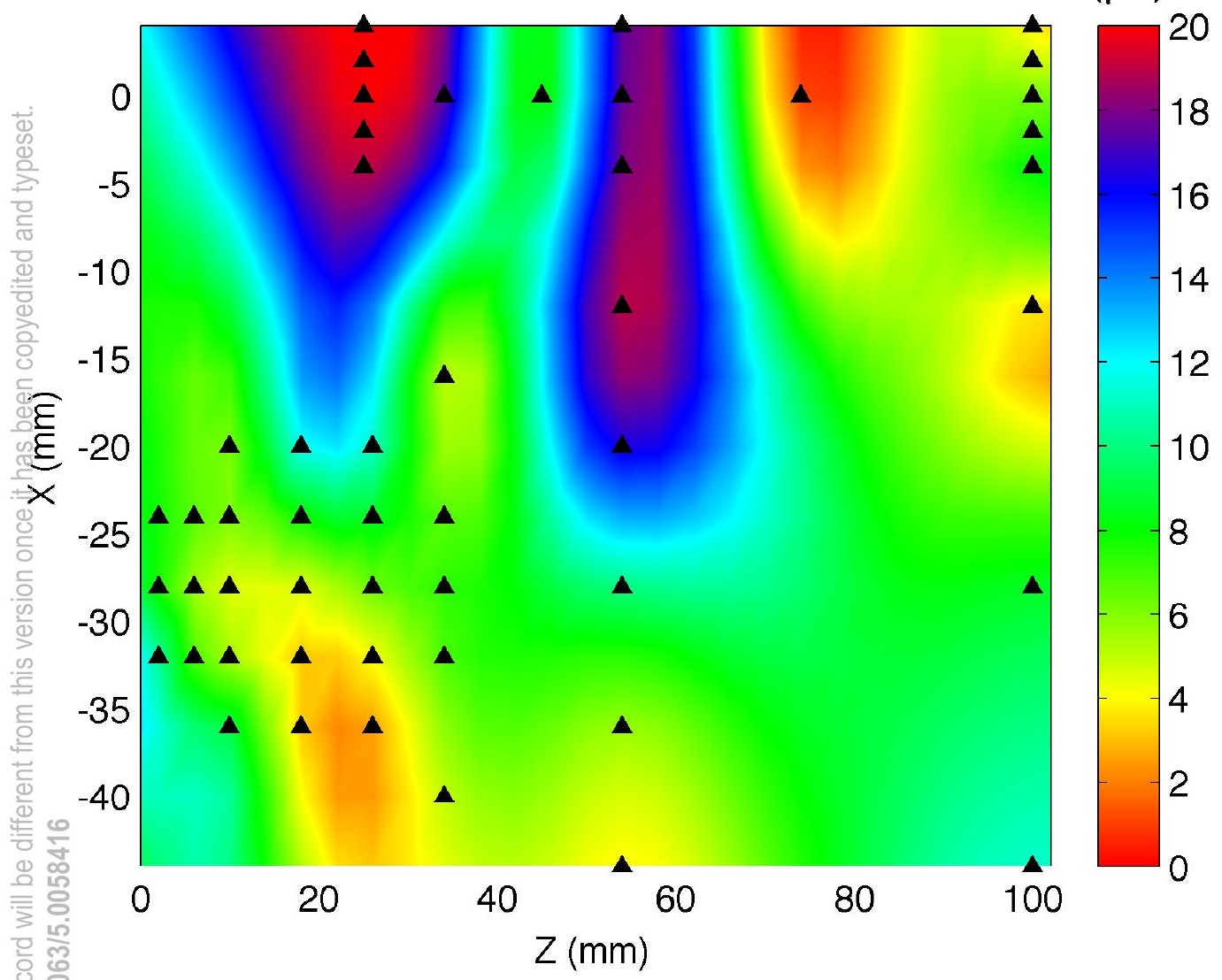

4)

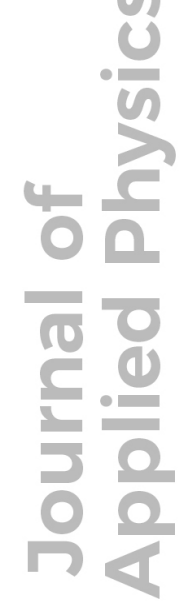

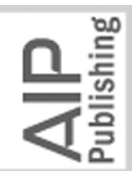




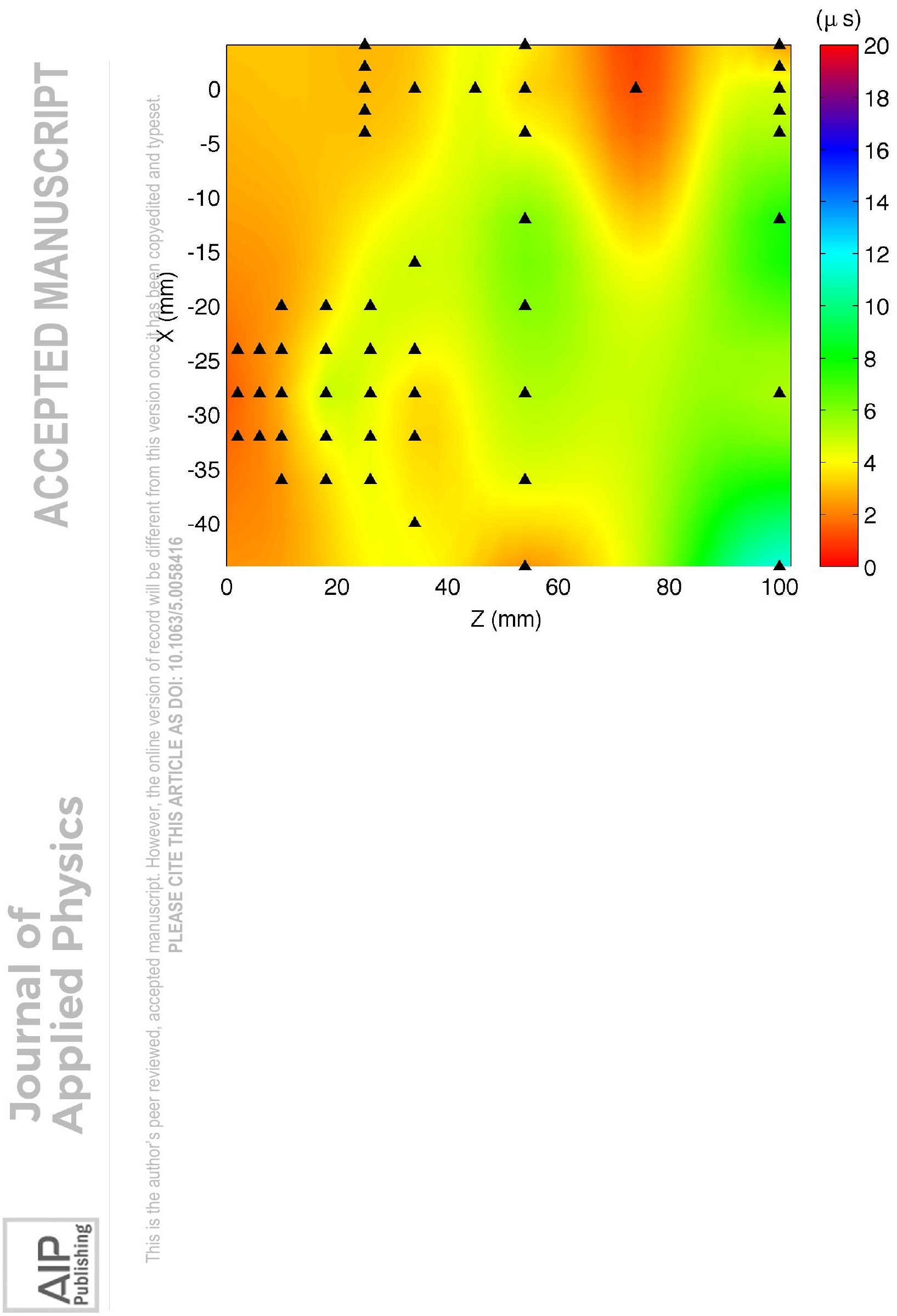




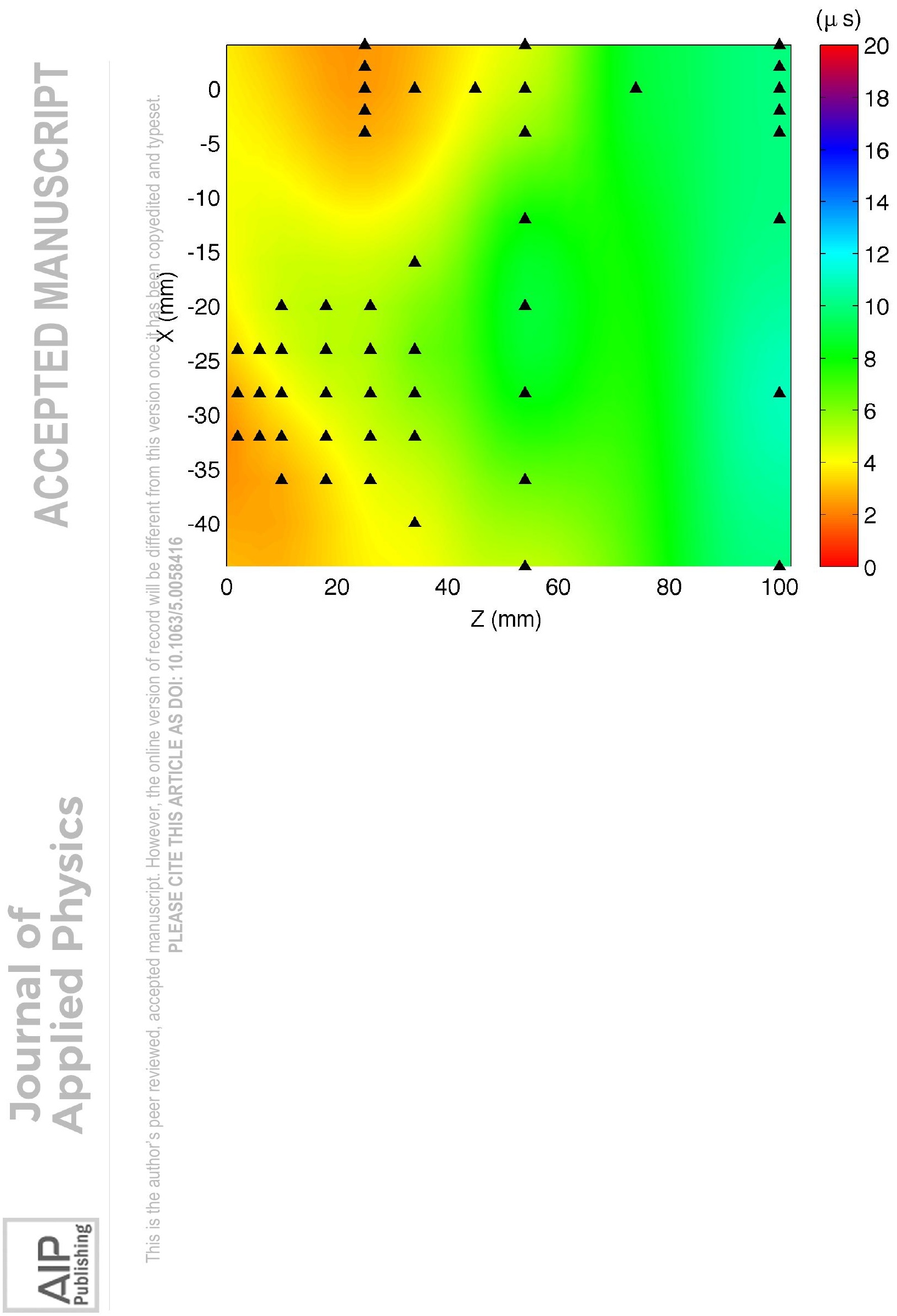


$x=-4 m m$
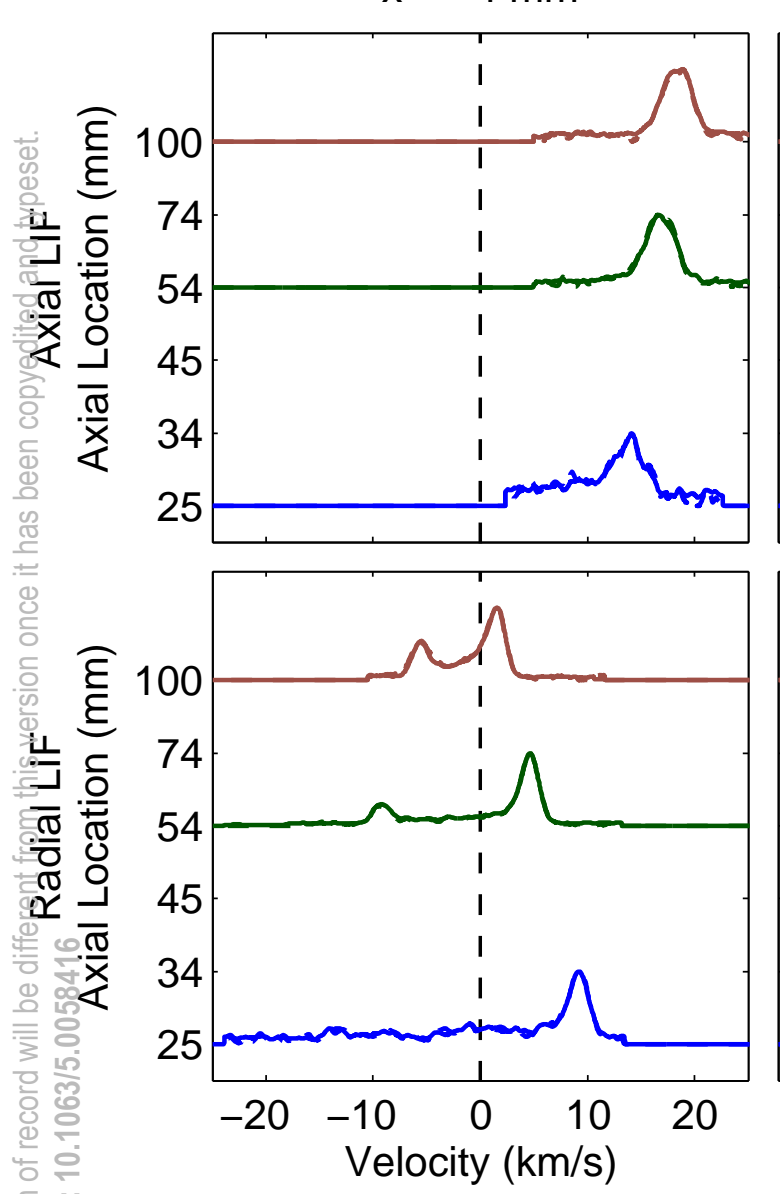
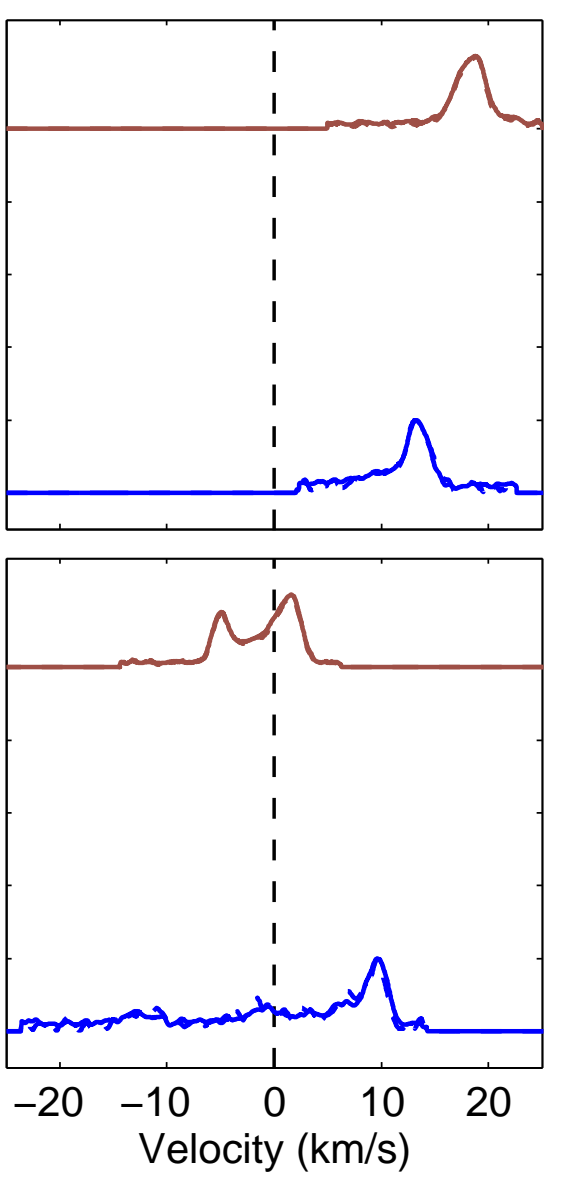
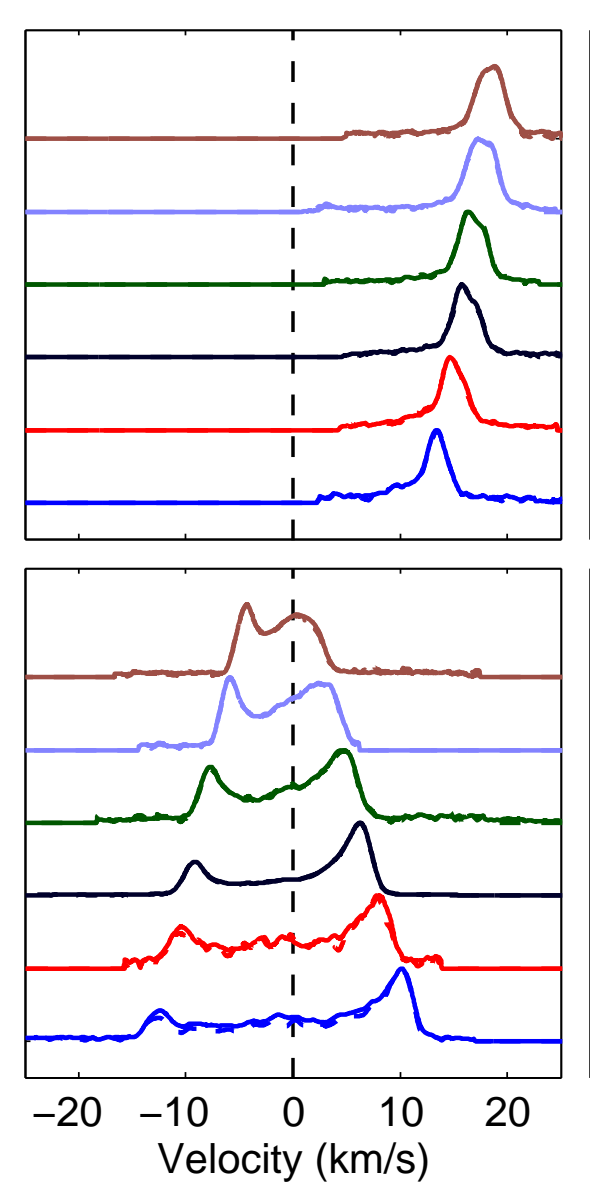

$x=2 \mathrm{~mm}$
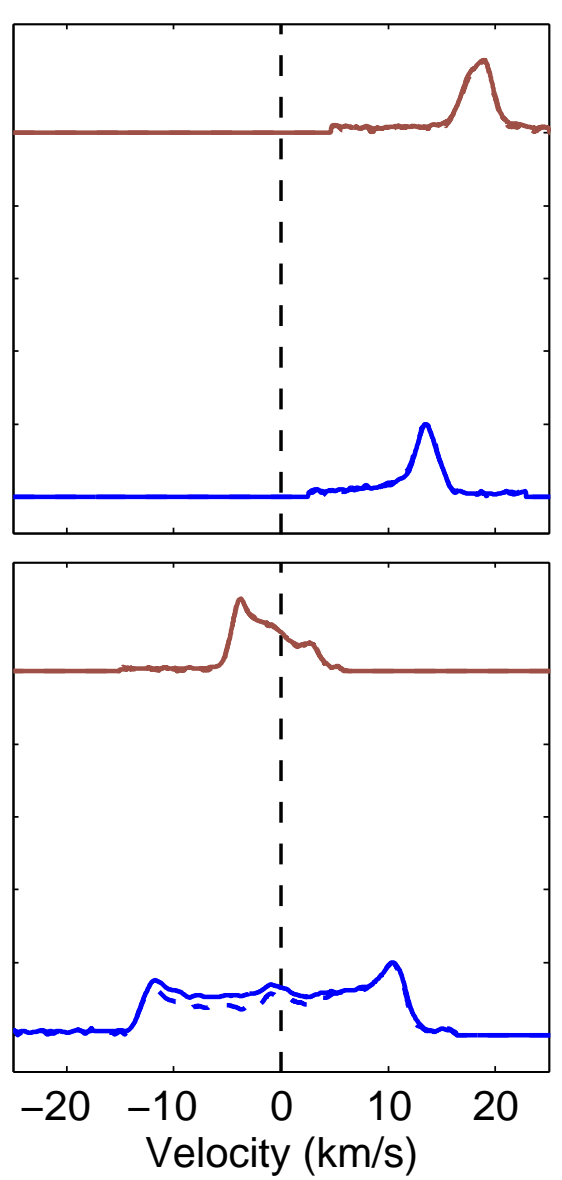

$\mathrm{x}=4 \mathrm{~mm}$

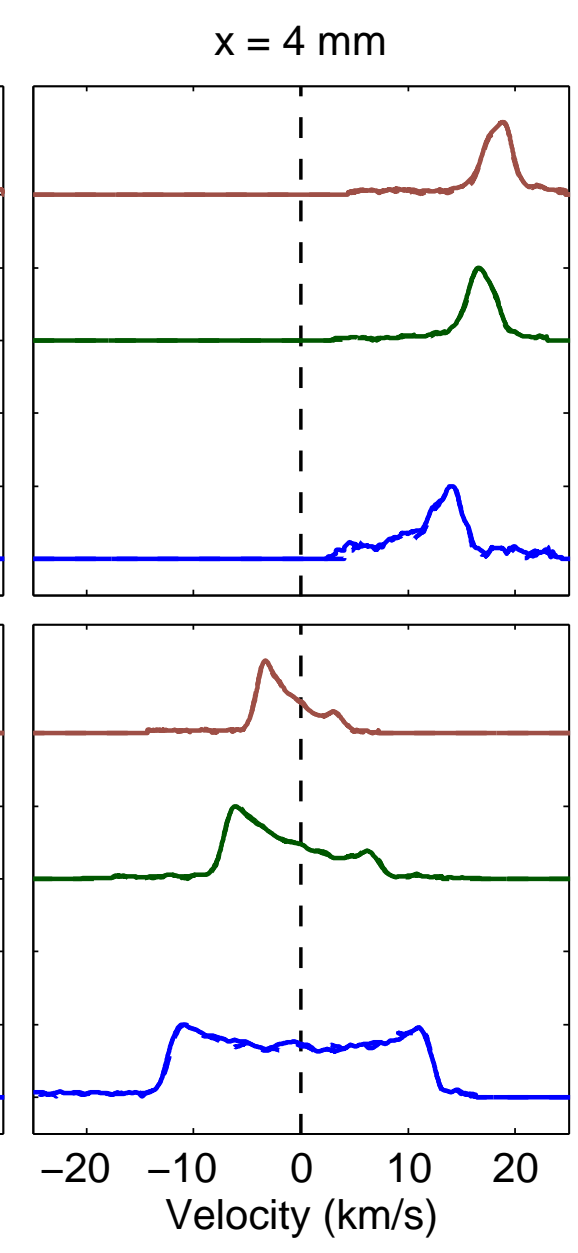




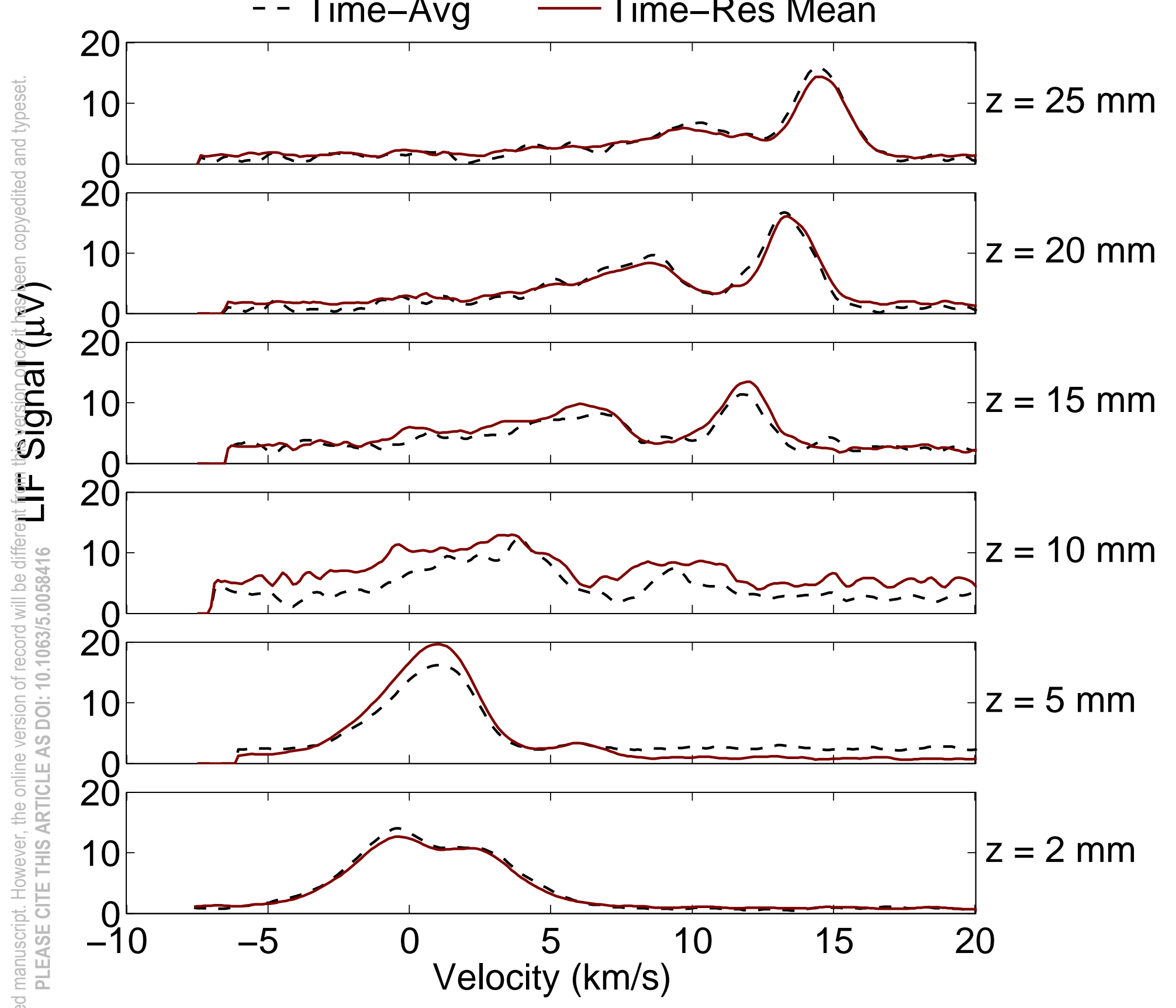




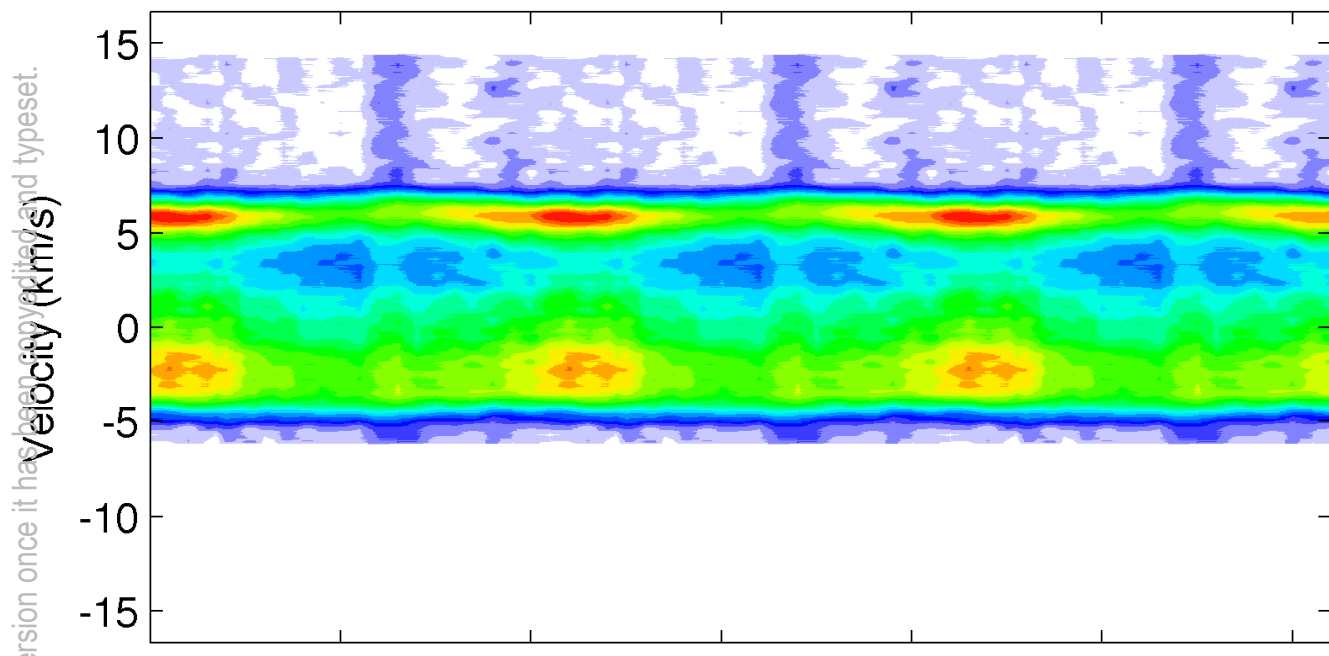$$
4
$$

这 3

$-$

4
3
0

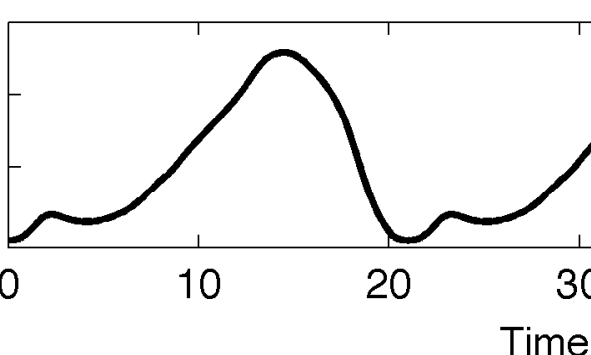

Time $(\mu s)$

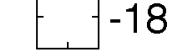



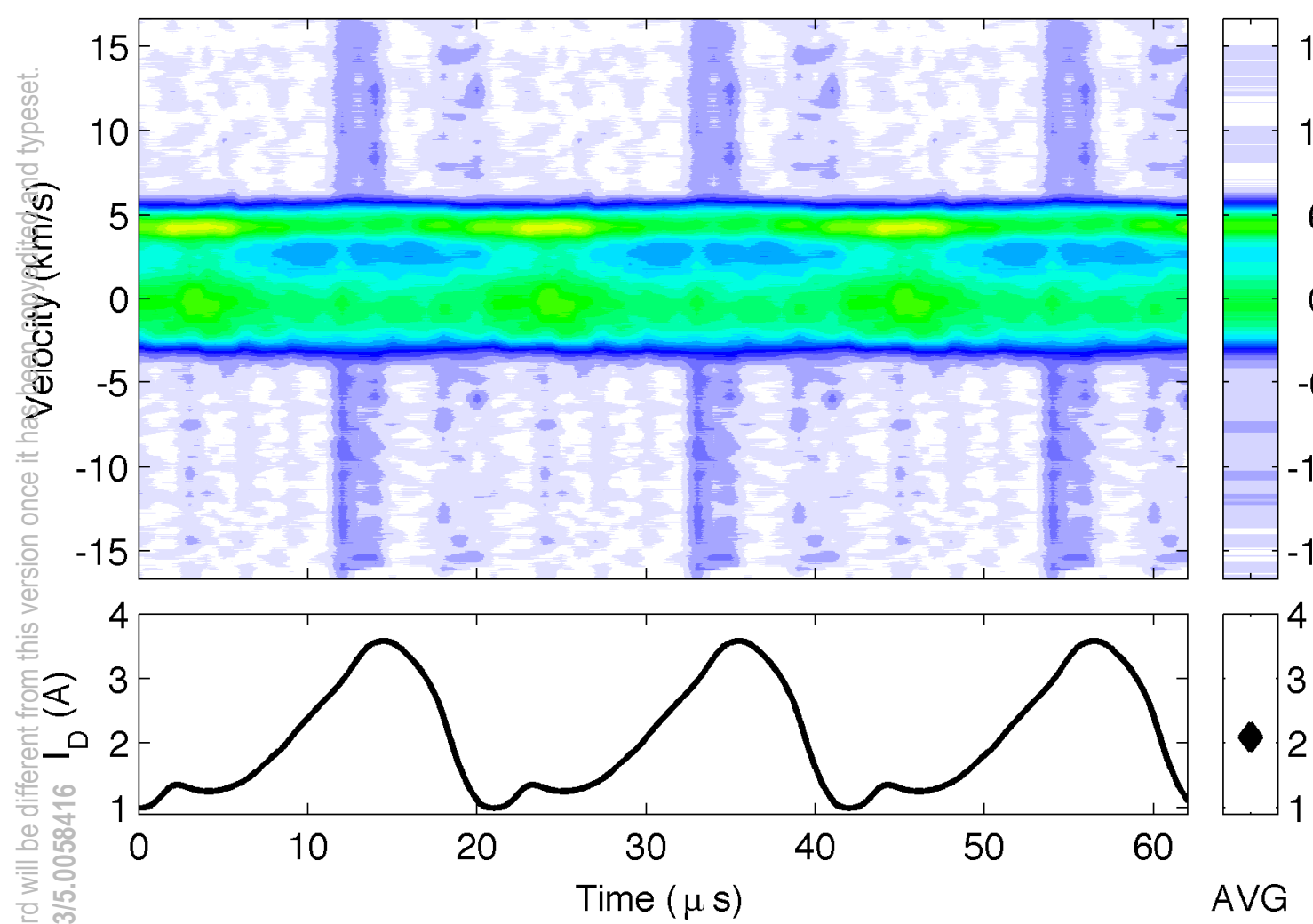
0.2

0.4

0.6

0.8

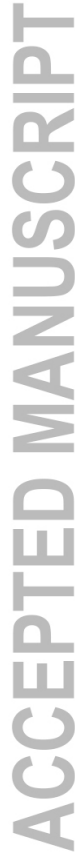
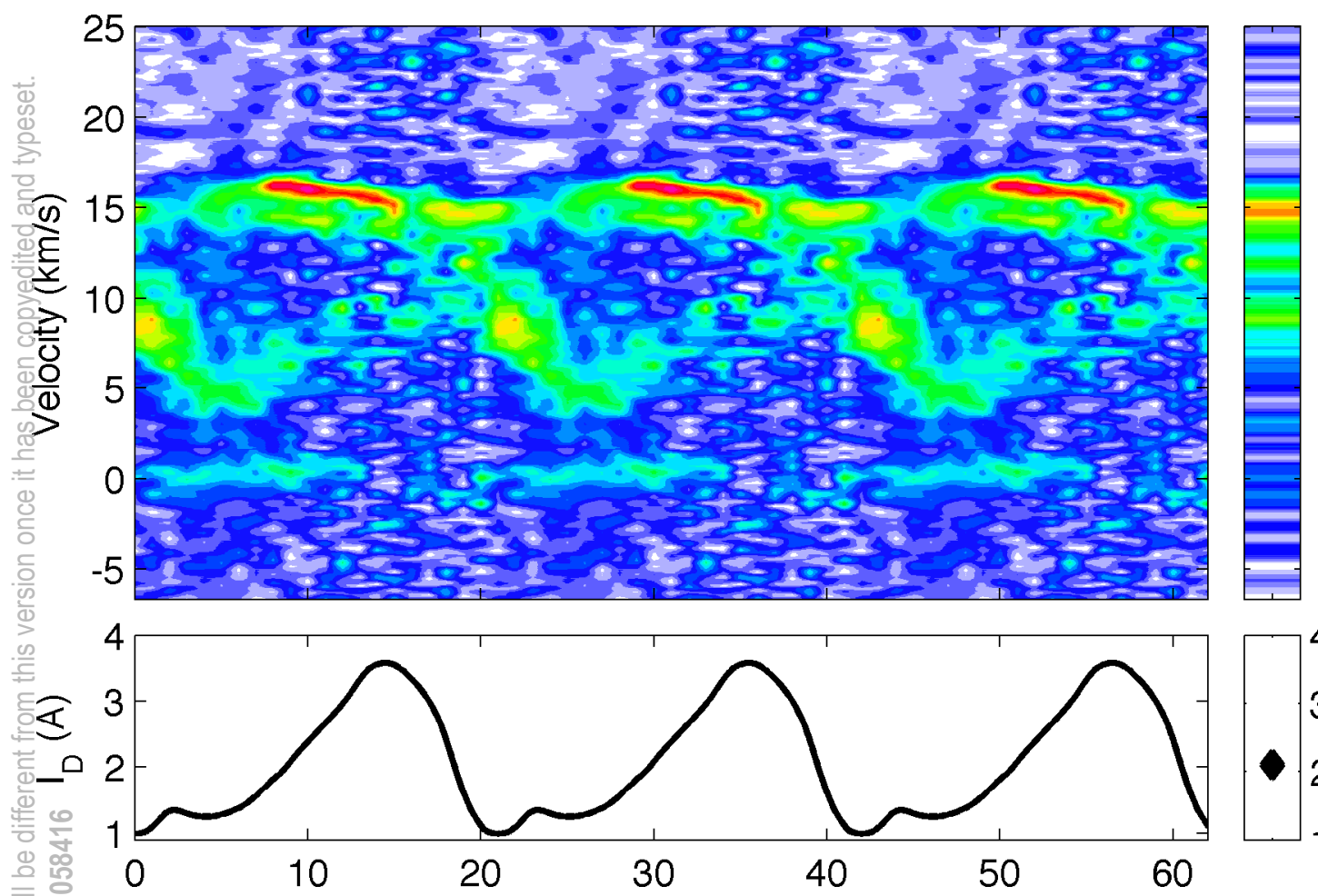

음

등 


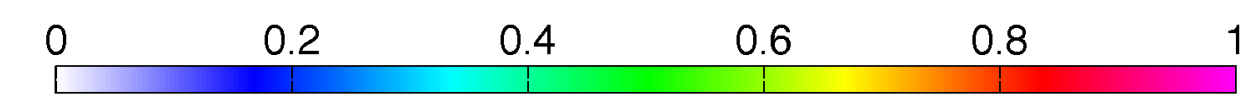

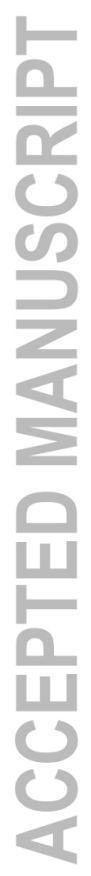

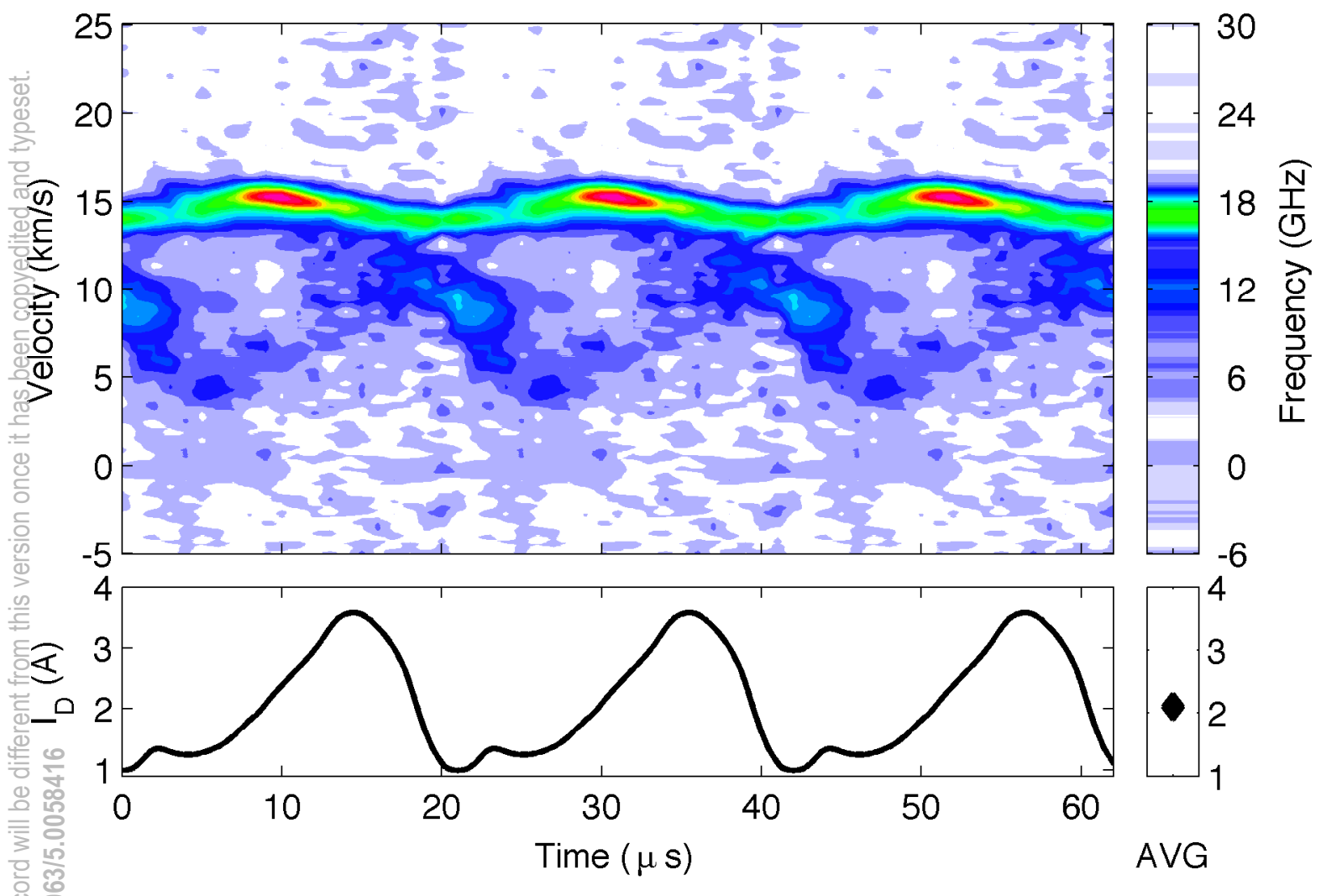

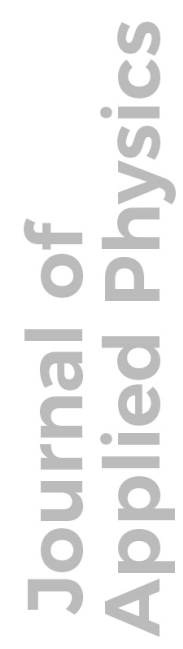

碞衰 
0.2

0.4

0.6

0.8

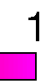

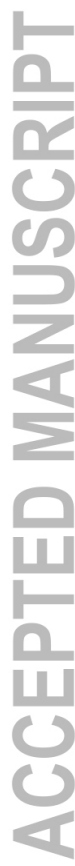

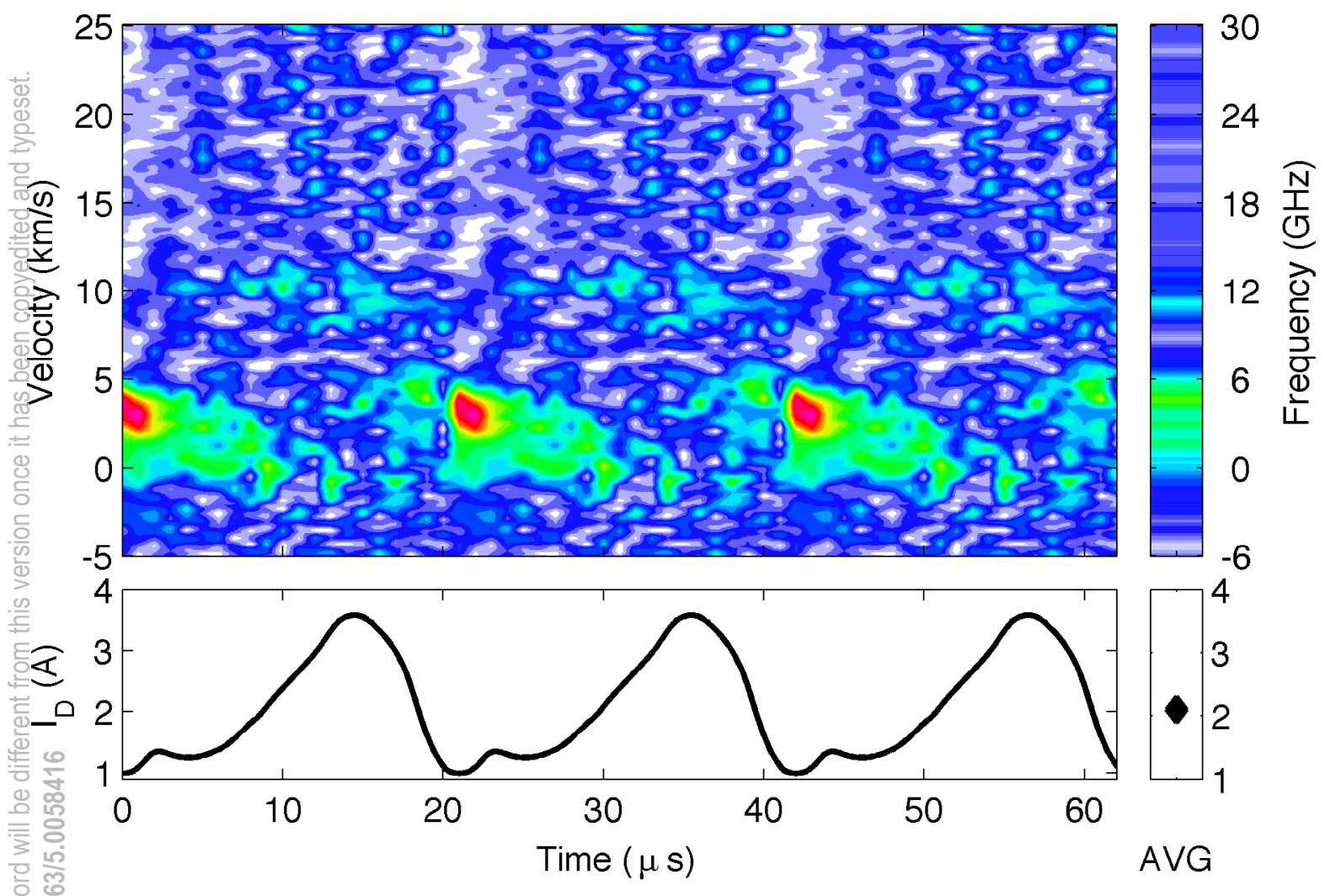

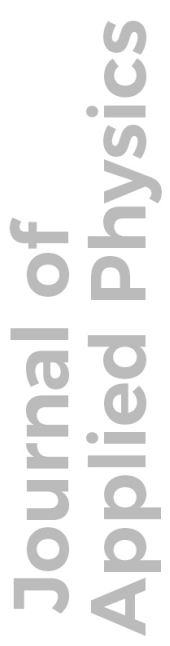

은 


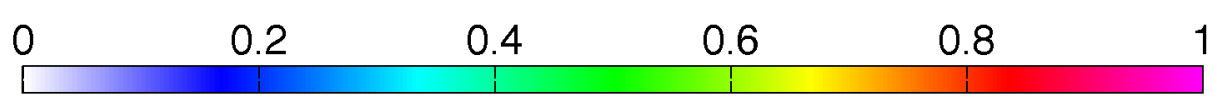

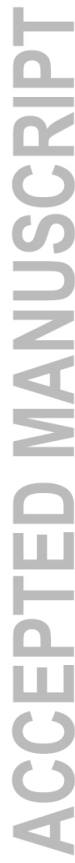

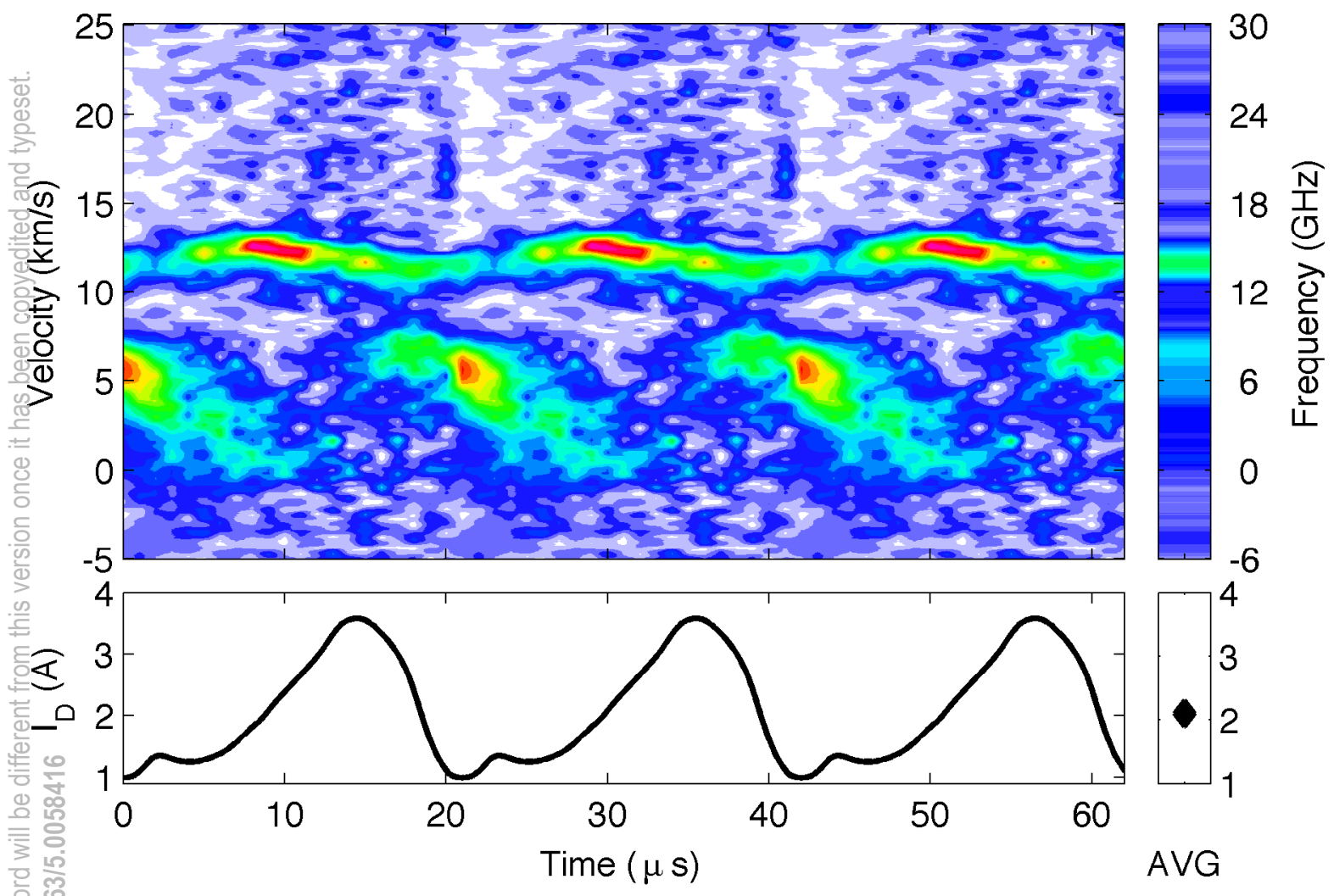

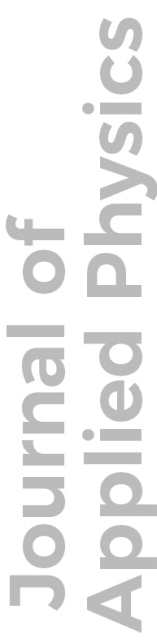

은 


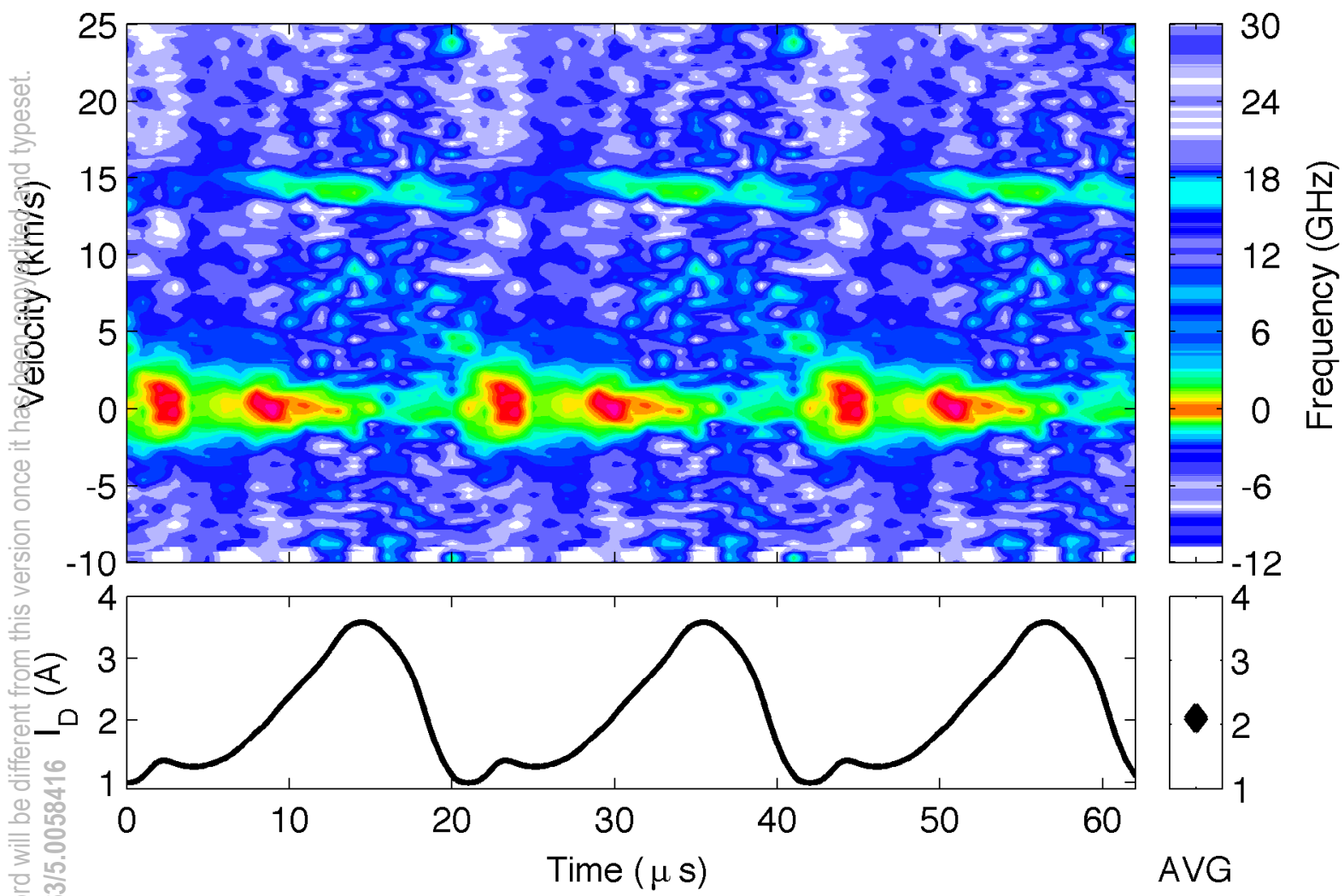



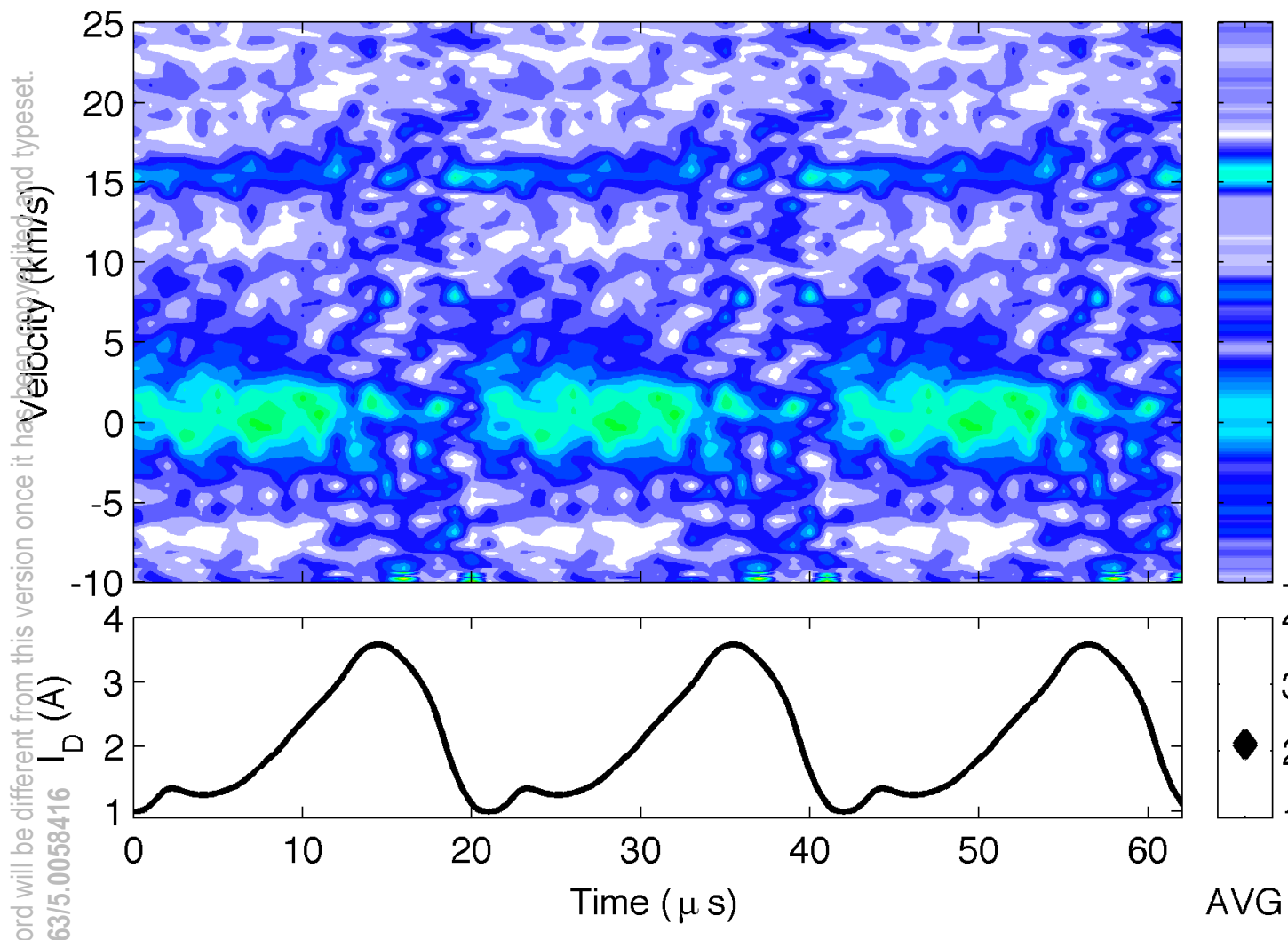

$\begin{array}{rr}18 & \frac{\widehat{N}}{N} \\ 12 & \frac{\mathrm{J}}{\mathrm{O}} \\ 6 & \frac{\mathrm{D}}{\mathrm{D}} \\ 0 & \frac{\mathrm{d}}{4}\end{array}$

$-6$

$-12$

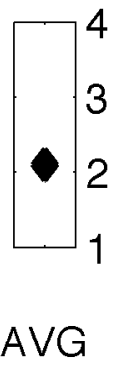

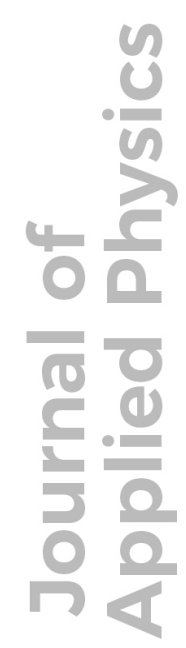

는 
